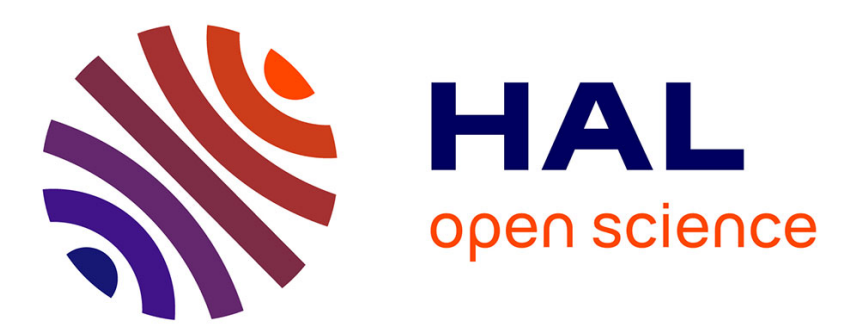

\title{
A Four-limb Parallel Schönflies Motion Generator with Full-circle End-effector Rotation
}

\author{
Guanglei Wu, Zirong Lin, Wenkang Zhao, Sida Zhang, Huiping Shen, \\ Stéphane Caro
}

\section{- To cite this version:}

Guanglei Wu, Zirong Lin, Wenkang Zhao, Sida Zhang, Huiping Shen, et al.. A Four-limb Parallel Schönflies Motion Generator with Full-circle End-effector Rotation. Mechanism and Machine Theory, 2020, 146, pp.103711. 10.1016/j.mechmachtheory.2019.103711 . hal-02373116

\section{HAL Id: hal-02373116 https://hal.science/hal-02373116}

Submitted on 20 Nov 2019

HAL is a multi-disciplinary open access archive for the deposit and dissemination of scientific research documents, whether they are published or not. The documents may come from teaching and research institutions in France or abroad, or from public or private research centers.
L'archive ouverte pluridisciplinaire HAL, est destinée au dépôt et à la diffusion de documents scientifiques de niveau recherche, publiés ou non, émanant des établissements d'enseignement et de recherche français ou étrangers, des laboratoires publics ou privés. 


\title{
A Four-limb Parallel Schönflies Motion Generator with Full-circle End-effector Rotation
}

\author{
Guanglei Wu, Zirong Lin, Wenkang Zhao, Sida Zhang \\ School of Mechanical Engineering, Dalian University of Technology, 116024 Dalian, China \\ E-mail: gwu@dlut.edu.cn \\ Huiping Shen \\ School of Mechanical Engineering, Changzhou University, 213100 Changzhou, China \\ E-mail: shp65@126.com \\ Stéphane Caro \\ CNRS, Laboratoire des Sciences du Numérique de Nantes \\ UMR CNRS 6004, Ecole Centrale de Nantes, 44321 Nantes, France \\ E-mail: stephane.caro@ls2n.fr
}

\begin{abstract}
This paper presents a four-limb parallel Schönflies motion generator for the pick-and-place application, whose end-effector is composed of a planetary gear train as the amplification mechanism to realize the full-circle rotation. The kinematic aspects including the workspace, dexterity and singularity are analyzed and evaluated. The singular configurations and the singularity loci are identified both graphically and numerically, which shows that the singular configurations of the manipulator can be avoided by keeping all the limbs working in a prescribed working mode together with the end-effector rotation in a clockwise direction from the neutral orientation. Moreover, the dexterity evaluation is carried out to depict the workspace quality and dexterous working envelope. It turns out that the proposed robot admits a super-ellipsoidal workspace with the fullcircle rotation of the end-effector, suitable for pick-and-place operations. Finally, robot dynamics is considered to select the actuators.
\end{abstract}

Keywords: Schönflies motion generator, parallel robot, pick-and-place applications, planetary gear train, singularity

\section{Introduction}

The parallel Schönflies motion generators (SMGs), which can produce three independent translations and one rotation about an axis of fixed direction, i.e., SCARA (Selective Compliance Assembly Robot Arm) motion, dedicate to the material handling for pick-and-place (PnP) applications, thanks to their advantages of high speed/stiffness and lightweight architecture. Up to date, numerous parallel SMGs have been developed. Amongst them, a number of four-limb parallel SMGs inherit the architecture of the H4 [1] robot that was created by the Pierrot's group from LIRMM, with four identical limbs and an articulated traveling plate [2]. Later on, the same research group developed the I4 [3], the symmetrical Par4 [4] and the Heli4 robots [5]. The latter two counterparts both have been commercialized as the Adept Quattro and the Veloce. robots, respectively. Four-limb parallel SMGs with Quattro architecture [6-11] can be found in the literature. Besides, other parallel SMGs are also noticeable for their high performance [12-18], such as the Delta based robots [19] and the two-limb SMGs [20-25], etc. 
Compared to the isostatic two-limb parallel SMGs $[20,21,26]$, the four-limb robot counterparts are hyperstatic. Amongst the existing four-limb SMGs, robots with a single platform [6,9,11] can significantly decrease the structural complexity towards low manufacturing cost. Those robots are suitable for the $\mathrm{PnP}$ applications that do not require a high rotational capability, due to their limited end-effector rotation without crossing any singular configuration. For instance, the small orientation angle of the robot end-effector will be applicable if the objects to be picked have a symmetrical shape. On the other hand, when the objects have irregular shapes, the robot end-effector with full-circle rotation will be required to accomplish the PnP task. In addition, design of parallel SMGs considers high end-effector rotational capability for extended application fields, for instance, the two five-bar mechanisms based parallel SCARA robot featuring infinite tool rotation for machining process [27]. The rotation of the parallel SMGs, such as H4, Par4 and Quattro, is realized by the relative movement in parallel between the two sub-platforms in their double-platform architecture, and then is amplified by the pulley-belt mechanism [4]. Different amplification mechanisms, i.e., the rack-pinion mechanism [3], gears and screw mechanism [5], have been adopted to enhance the rotational capability, of which the size and shape will be changed when the previous robot end-effectors are in different orientations. This may not be advantageous to the requirements of robot end-effectors, e.g., being easy to be cleaned, particularly for food industry-a major sector of industrial applications. In this work, a parallel SMG, whose end-effector adopts a planetary gear train (PGT) as the amplification mechanism to realize the full-circle rotation, is proposed. The whole transmitting amplification mechanism can be sealed in a gearbox, which is suitable for food robotics. The robot performances, such as the workspace, dexterity, singularity and dynamics, are to be investigated to evaluate the robot design.

In this paper, a four-limb parallel SMG with full-circle rotation is proposed, of which the planetary gear train (PGT) is adopted to construct the mobile platform. The mobilities of the proposed robot is verified with the displacement group. The kinematic aspects of the robot, namely, position analysis, workspace, dexterity and singularity are analyzed together with dynamics. The results show that the proposed robot admits a singularity-free workspace with the full-circle rotation of the end-effector, suitable for pick-and-place operations.

\section{Manipulator Architecture}

Figure 1 depicts the simplified CAD model of the design of the new four-limb robot. Similar to its existing robot counterparts, each limb is composed of an actuated proximal link and a passive distal arm (parallelogram, a.k.a " $\Pi$ joint") connected to the base and mobile platforms, as shown in Fig. 1(a). The geometric centers of the four actuated joints are located at the vertices of a rectangle on the base platform to avoid the singularities when the robot reaches some symmetric configurations.

As displayed in Fig. 1(b), the mobile platform is consisted of a constant-orientation sub-platform as the gear ring and a rotational sub-platform as the carrier in the PGT. Both sub-platforms connect a pair of opposite limbs, where the rotational one has two additional revolute pairs at the connecting positions rather than the remaining limbs. The end-effector orientation is amplified by the PGT, which can be simplified as a revolute pair. The corresponding joint-loop graph is shown in Fig. 1(c). The orientation of the rotational sub-platform is hence can be computed from the required end-effector orientation and speed ratio of the PGT below

$$
i_{e H}=\frac{\omega_{e}}{\omega_{H}}=1+\frac{z_{r}}{z_{e}}
$$

where $\omega_{e}$ and $\omega_{H}$ stand for the angular velocities of the output end-effector and the rotational subplatform, respectively. Moreover, $z_{r}$ and $z_{e}$ are the number of the teeth of the gear ring and the output 


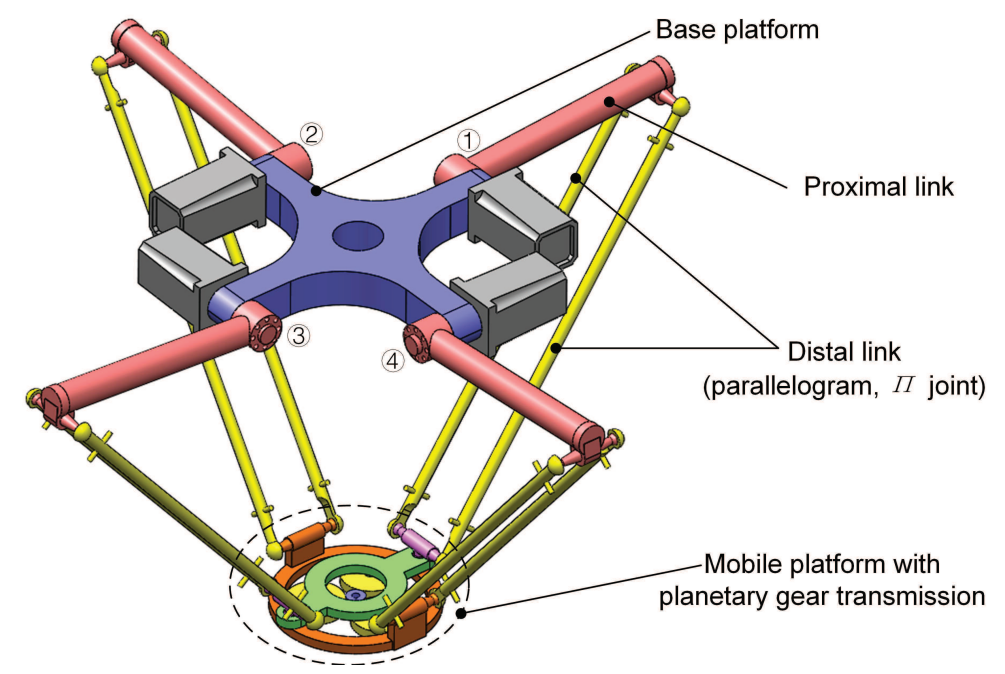

(a)

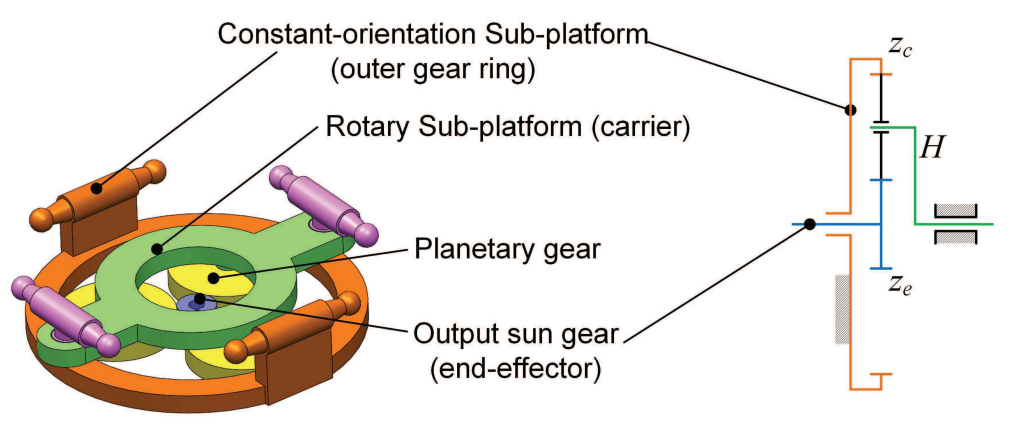

(b)

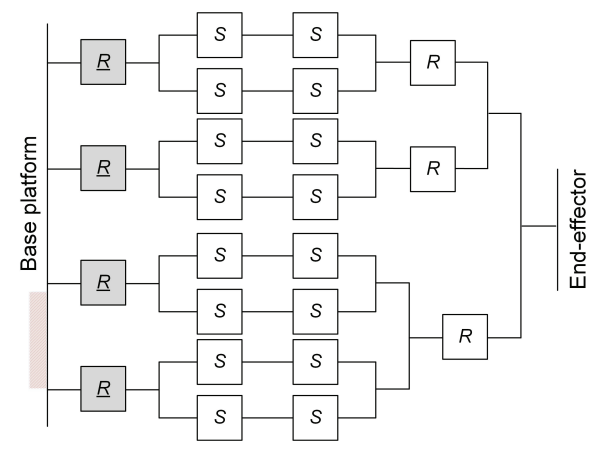

(c)

Figure 1: The CAD model of of the new SMG: (a) overall architecture; (b) mobile platform composed of planetary gear train; (c) joint-and-loop graph, where gray $\mathrm{R}$ joints stand for active joints and remaining $\mathrm{R}$ and $\mathrm{S}$ joints stand for the passive joints without the amplification mechanism.

sun gear, respectively. One example is that $i_{e H}=8$ with $z_{r}=140$ and $z_{e}=20$ to realize the full-circle end-effector rotation with the sub-platform rotation $45^{\circ}$.

\section{Displacement Group and Mobility Analysis}

The mobility of the proposed SMG is analyzed by the theory of Lie group of rigid body displacements [28]. When all the limbs of the manipulator are assembled, the end-effector mobilities will be the ones that is allowed by every limb, i.e., the intersection of the kinematic bonds generated by all the limbs. Prior to derivation, some displacement sets [15] are defined below:

$\{\mathcal{R}(N, \mathbf{u})\}$ 1-D rotational motion subgroup defined by a point $N$ located in its axis of rotation and a unit vector $\mathbf{u}$ defining its direction

$\{\mathcal{T} 2(\mathbf{u})\} 2$-D translational group lying in a plane that is normal to a unit vector $\mathbf{u}$

$\{\mathcal{T}\}$ 3-D translational group

$\{\mathcal{X}(\mathbf{u})\}$ Schönflies motion subgroup with unit vector $\mathbf{u}$ defining the direction of its rotational axis 
The robot is composed of two $R R \Pi R R$ and two $R R \Pi R$ limb types as shown in Fig. 2 and has two symmetrical planes $O x z$ or $O y z$. Let $\mathbf{i}, \mathbf{y}$, and $\mathbf{k}$ denote the unit vectors along $x$-, $y$-, and $z$-axis, respectively, the kinematic bond $\mathcal{L}_{i}$ of the $i$ th limb, $i=2$, 4 , is the product of the following four bonds:

- The rotational subgroup $\mathcal{R}\left(A_{i}, \mathbf{i}_{A}\right)$ passing through point $A_{i}$ and parallel to the unit vector $\mathbf{i}$ of $x$-axis.

- The rotational subgroup $\mathcal{R}\left(B_{i}, \mathbf{i}_{B}\right)$ passing through point $B_{i}$ and parallel to $\mathbf{i}_{A}$.

- The 2-D translational subgroup $\mathcal{T} 2\left(\mathbf{n}_{i}\right)$ corresponding to the parallelogram that is characterized by translations around circles of radius $\overline{B_{i} C_{i}}$ lying in a plane normal to the vector $\mathbf{n}_{i}$.

- The rotational subgroup $\mathcal{R}\left(C_{i}, \mathbf{i}_{C}\right)$ passing through point $C_{i}$ and parallel to $\mathbf{i}_{B}$.

by contrast, the rotational subgroups passing through points $A_{i}, B_{i}$ and $C_{i}$ are parallel to the $y$-axis in the first and third limbs, and one more rotational subgroup $\mathcal{R}\left(C_{i}, \mathbf{k}\right)$ passing through point $C_{i}$ and parallel to $z$-axis exist. Thus, the kinematic bonds of the $i$ th limb is

$$
\begin{aligned}
& \mathcal{L}_{i}=\mathcal{R}\left(A_{i}, \mathbf{j}_{A}\right) \cdot \mathcal{R}\left(B_{i}, \mathbf{j}_{B}\right) \cdot \mathcal{T} 2\left(\mathbf{n}_{i}\right) \cdot \mathcal{R}\left(C_{i}, \mathbf{j}_{C}\right) \cdot \mathcal{R}\left(C_{i}, \mathbf{k}\right)=\mathcal{X}(\mathbf{j}) \cdot \mathcal{R}(\mathbf{k}), i=1,3 \\
& \mathcal{L}_{i}=\mathcal{R}\left(A_{i}, \mathbf{i}_{A}\right) \cdot \mathcal{R}\left(B_{i}, \mathbf{i}_{B}\right) \cdot \mathcal{T} 2\left(\mathbf{n}_{i}\right) \cdot \mathcal{R}\left(C_{i}, \mathbf{i}_{C}\right)=\mathcal{X}(\mathbf{i}), i=2,4
\end{aligned}
$$

thus, the intersections of the leg pairs 1-3 and 2-4 are expressed as

$$
\begin{aligned}
& \mathcal{L}_{13}=\mathcal{X}(\mathbf{j}) \cdot \mathcal{R}(\mathbf{k})=\mathcal{T} \cdot \mathcal{R}(\mathbf{j}) \cdot \mathcal{R}(\mathbf{k}), i=1,3 \\
& \mathcal{L}_{24}=\mathcal{X}(\mathbf{i})=\mathcal{T} \cdot \mathcal{R}(\mathbf{i}), i=2,4
\end{aligned}
$$

As two sub-platforms constrained by leg pairs 1-3 and 2-4 are connected serially via a rotational subgroup $\mathcal{R}(P, \mathbf{k})$, the subgroup of the robot end-effector is hence equal to

$$
\mathcal{L}=\mathcal{L}_{13} \cap \mathcal{L}_{24} \cdot \mathcal{R}(P, \mathbf{k})=\mathcal{T} \cdot \mathcal{R}(\mathbf{k}) \equiv \mathcal{X}(\mathbf{k})
$$

To this end, the intersection of all subgroups is a Schönflies subgroup $\mathcal{X}(\mathbf{k})$, meaning that the robot generates the Schönflies motion.

\section{Robot Geometry and Kinematic Jacobian Matrix}

The parametrization of the robot is shown in Fig. 3(a). The reference coordinate frame $\mathcal{F}_{b}$ is built with the origin located at the geometric center $O$ of the base platform, with $x$-axis being parallel to the segment $A_{2} A_{1}\left(A_{3} A_{4}\right)$. The moving coordinate frame $\mathcal{F}_{p}$ is attached to the rotational sub-platform with the origin located at the geometric center $P$, and its $x$-axis is parallel to segment $C_{3} C_{1}$. The base platform dimension is depicted by the radius $R$ with two arc centers shifted by distance $2 a$ along $y$-axis, and the mobile platform dimension is depicted by radius $r$ that is equal to the length of segment $\overline{P C_{i}}$. The segments $\overline{A_{i} B_{i}}=b$ and $\overline{B_{i} C_{i}}=l$ stand for the lengths of the proximal and distal links, respectively. In the $i$ th limb, the axis of rotation of the actuated joint is parallel to unit vector $\mathbf{u}_{i}$, and unit vectors $\mathbf{v}_{i}$ and $\mathbf{w}_{i}$ are parallel to segments $A_{i} B_{i}$ and $B_{i} C_{i}$, respectively.

With the unit vector defined in last section, the Cartesian coordinates of point $A_{i}$ in the frame $\mathcal{F}_{b}$ are denoted as

$$
\mathbf{a}_{i}=\left\{\begin{array}{l}
R \mathbf{e}_{i}+a \mathbf{j}, i=1,2 \\
R \mathbf{e}_{i}-a \mathbf{j}, i=3,4
\end{array}\right.
$$

with

$$
\mathbf{e}_{i}=\left[\begin{array}{lll}
\cos \alpha_{i} & \sin \alpha_{i} & 0
\end{array}\right]^{T}, \alpha_{i}=\frac{2 i-1}{4} \pi
$$




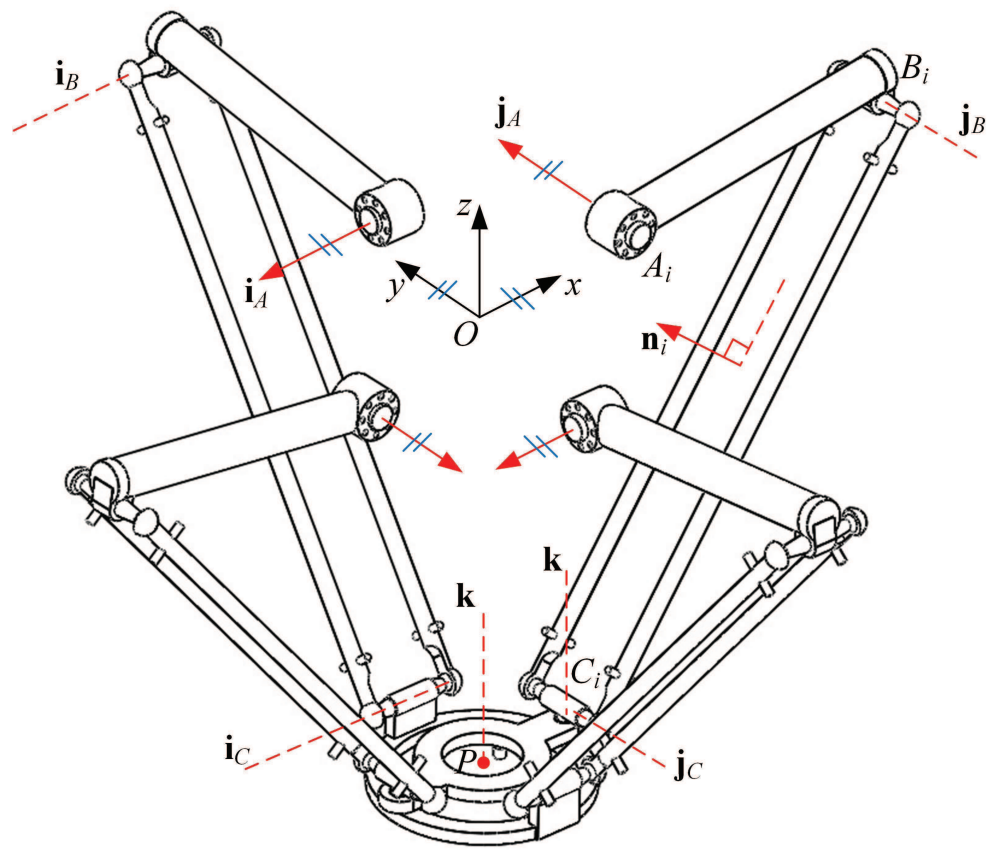

Figure 2: The robot architecture with axes of rotation at points $A_{i}, B_{i}$ and $C_{i}$.

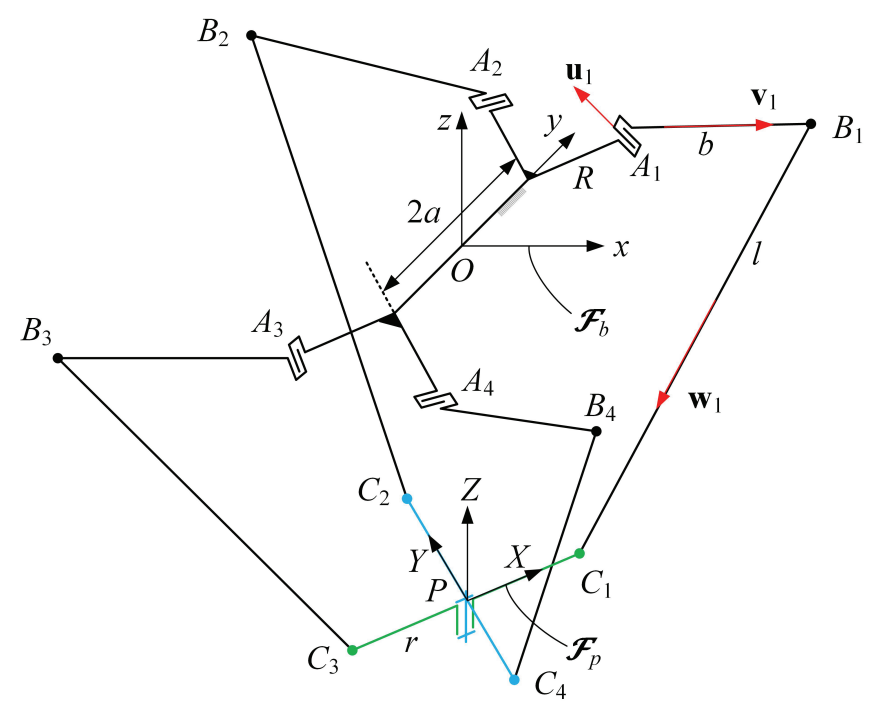

(a)

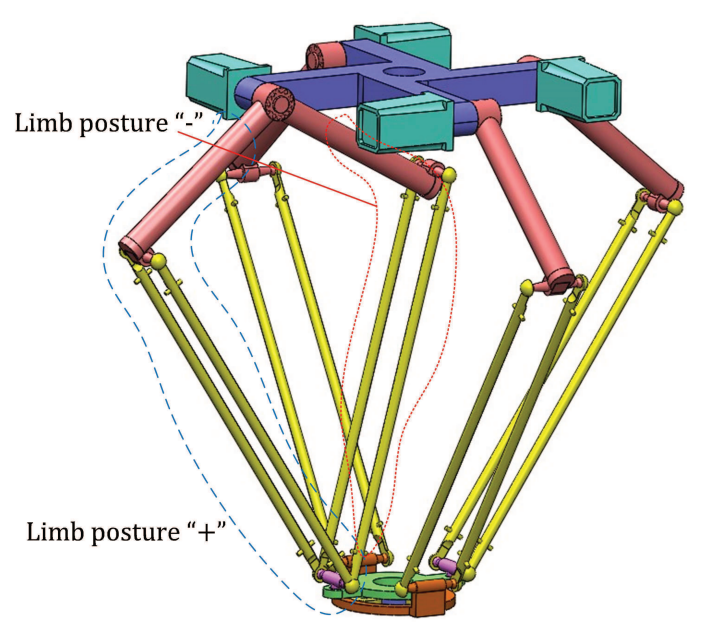

(b)

Figure 3: (a) Parameterization of the robot architecture and (b) two postures of one limb. 
and the Cartesian coordinates of point $B_{i}$ in the frame $\mathcal{F}_{b}$ are given by

$$
\mathbf{b}_{i}=b \mathbf{v}_{i}+\mathbf{a}_{i}, \mathbf{v}_{i}=\mathbf{R}_{z}\left(\alpha_{i}\right) \mathbf{R}_{y}\left(\theta_{i}\right) \hat{\mathbf{x}}
$$

with the corresponding axis of rotation of the $i$ th actuated joint is described by $\mathbf{u}_{i}=\left[\begin{array}{lll}-\sin \alpha_{i} & \cos \alpha_{i} & 0\end{array}\right]^{T}$.

Let the end-effector pose be $\boldsymbol{\chi}=\left[\begin{array}{ll}\mathbf{p}^{T} & \phi\end{array}\right]^{T}, \mathbf{p}=\left[\begin{array}{lll}x & y & z\end{array}\right]^{T}$, the Cartesian coordinates of point $C_{i}$ in frame $\mathcal{F}_{b}$ is expressed as

$$
\mathbf{c}_{i}=\left\{\begin{array}{l}
r \mathbf{Q} \mathbf{i}+\mathbf{p}, i=1,3 \\
r \mathbf{R}_{z}\left(\alpha_{i}\right) \mathbf{i}+\mathbf{p}, i=2,4
\end{array}\right.
$$

where $\mathbf{Q}=\mathbf{R}_{z}\left(\phi+\phi_{0}+(i-1) \pi / 2\right)$ is the rotation matrix of the rotational sub-platform, and $\phi_{0}$ is the neutral orientation angle of the rotational sub-platform.

By making use of the following kinematic constraints:

$$
\left(\mathbf{c}_{i}-\mathbf{b}_{i}\right)^{T}\left(\mathbf{c}_{i}-\mathbf{b}_{i}\right)=l^{2}, \quad i=1, \ldots, 4
$$

the inverse geometry problem of the robot can be solved as

$$
\theta_{i}=2 \tan ^{-1} \frac{-I_{i} \pm \sqrt{I_{i}^{2}+J_{i}^{2}-K_{i}^{2}}}{K_{i}-J_{i}}
$$

with

$$
I_{i}=-2 b\left(\mathbf{c}_{i}-\mathbf{a}_{i}\right)^{T} \mathbf{k}, J_{i}=2 b\left(\mathbf{c}_{i}-\mathbf{a}_{i}\right)^{T} \mathbf{e}_{i}, K_{i}=l^{2}-b^{2}-\left\|\mathbf{c}_{i}-\mathbf{a}_{i}\right\|^{2}
$$

For a given pose of the robot end-effector, each limb can have two postures that are characterized by the sign "-/+" in Eq. (10), which means that the robot can have up to 16 working modes ( $\mathcal{W} \mathcal{M}$ s). Here, the signs "+" and "-" stand for two different postures for each limb, respectively, as depicted in Fig. 3(b). To avoid the mechanical collision, the working mode corresponding to the four limbs in posture "+" is selected from the 16 working modes for this type of robot.

Differentiating the four Eqs. (9) with respect to time yields

$$
\mathbf{A} \dot{\chi}=\mathbf{B} \dot{\theta}
$$

with

$$
\mathbf{A}=\left[\begin{array}{cc}
\mathbf{w}_{1}^{T} & r \mathbf{w}_{1}^{T} \mathbf{s} \\
\mathbf{w}_{2}^{T} & 0 \\
\mathbf{w}_{3}^{T} & -r \mathbf{w}_{3}^{T} \mathbf{s} \\
\mathbf{w}_{4}^{T} & 0
\end{array}\right], \dot{\boldsymbol{\chi}}=\left[\begin{array}{c}
\dot{x} \\
\dot{y} \\
\dot{z} \\
\dot{\phi}
\end{array}\right] ; \mathbf{B}=\left[\begin{array}{llll}
h_{1} & & & \\
& h_{2} & & \\
& & h_{3} & \\
& & & h_{4}
\end{array}\right], \dot{\boldsymbol{\theta}}=\left[\begin{array}{c}
\dot{\theta}_{1} \\
\dot{\theta}_{2} \\
\dot{\theta}_{3} \\
\dot{\theta}_{4}
\end{array}\right]
$$

where

$$
\mathbf{s}=\left[\begin{array}{lll}
-\sin \left(\phi+\phi_{0}\right) & \cos \left(\phi+\phi_{0}\right) & 0
\end{array}\right]^{T} ; h_{i}=b\left(\mathbf{u}_{i} \times \mathbf{v}_{i}\right) \cdot \mathbf{w}_{i}, \mathbf{w}_{i}=\left(\mathbf{c}_{i}-\mathbf{b}_{i}\right) / l
$$

In Eq. (12), $\mathbf{A}$ and $\mathbf{B}$ are named as the forward and inverse Jacobian matrices, respectively. The kinematic Jacobian matrix is hence obtained as $\mathbf{J}=\mathbf{A}^{-1} \mathbf{B}$ as long as matrix $\mathbf{A}$ is not rank deficient.

\section{Performance Evaluation of the Proposed SMG}

The kinematic design of a parallel manipulator usually concerns the evaluation of the workspace, dexterity and singularity. The characteristics of the workspace that reflects the shape, size and presence of singularities are of primary importance. Workspace, in the context of this paper, refers to a singularity-free volume that the robot end-effector can reach with full-circle rotation. The dexterity is usually evaluated by means of the condition number of the kinematic Jacobian matrix. Based upon the design analysis in previous work [29], the geometric parameters of the proposed SMG in the unit of $\mathrm{mm}$, are set to $a=100, R=150, b=300, l=600, r=100$, respectively. 


\subsection{Singularity Analysis}

In the design and application of robotics, singularity analysis is a central issue due to their major effect on the robot performance. A common approach to identify the singularity is to examine the determinants of the kinematic Jacobian matrices of the manipulator [30]. Whereas, this analysis method cannot find the constraint singularity [31] that occurs in the restricted motion. Alternatively, the constraint singularity can be analyzed from the motion/force constraint performance, utilizing the approaches of Grassmann line geometry [32,33] and screw theory [12].

\subsubsection{Jacobian analysis based singularity}

It is known that the manipulator is in a singular configuration when the determinant of its forward or/and the determinant of its inverse Jacobian matrix is (are) equal to zero [30], namely,

- Type-1 singularity, also named as limb singularity, occurs when $|\mathbf{B}|=0^{1}$.

- Type-2 singularity, also named as actuation singularity, occurs when $|\mathbf{A}|=0$.

- Third type of singularities can be identified when both Type-1 and Type-2 singularities occur simultaneously.

From $|\mathbf{B}|=\prod_{i=1}^{4} h_{i}=b^{4} \prod_{i=1}^{4}\left(\mathbf{u}_{i} \times \mathbf{v}_{i}\right)^{T} \mathbf{w}_{i}$, the type-1 singularities occur when the actuated link $A_{i} B_{i}$ and the segment of parallelogram $B_{i} C_{i}$ are coplanar, usually existing at the reachable workspace boundaries, which can be readily identified. Therefore, the type-1 singularity will be briefly discussed.

When the determinant $|\mathbf{A}|$ of the forward Jacobian matrix is equal to zero, the actuation singularity occurs in the case of the general linear complex. The wrench exerting on the end-effector is generated by one of the transmission wrenches that is proportional to those that are generated by the other transmission wrenches, thus, the robot loses 1-DOF motion in this singular configuration [34].

Type-1 singularity loci The type-1 singularity loci, which can be readily identified, are located on the reachable workspace boundaries [34]. Figure 4(a) displays all the possible type-1 singularity loci of limb 1 with constant end-effector orientation $\phi=0$, which are subject to the motion range of the actuated joints. The type- 1 singularity loci of the remaining limbs are symmetrical to limb 1 singularity loci with respect to planes $x z$ and $y z$. Sequentially, the maximum regular workspace free of type-1 singularity and mechanical collision can be found as shown in Fig. 4(b). Figure 4(c) displays the regular workspace with different end-effector orientations, from which it is seen that the workspace volume free of type-1 singularities decreases with the increasing end-effector orientation.

Type-2 singularity loci As the expression of the determinant $|\mathbf{A}|$ of the forward Jacobian matrix is a complex and implicit equation, the singularity loci cannot be detected by means of the discrete searching method. The workspace is described with small increments to check $|\mathbf{A}|$ for different constant end-effector orientations. If $|\mathbf{A}|\left\langle\Delta, \Delta=10^{-3}\right.$ being a computational tolerance, the robot can be considered in a singular configuration. Moreover, interpolation is introduced to smooth the singularity surface. Figure 5 shows the type-2 singularity loci with end-effector orientations $\phi=0$ and $\phi=-5^{\circ}$, together with the fitted regular workspace. It is observed that the singular postures reduce when the amplitude of the end-effector orientation increases. Since the pick-and-place operations are usually confined in a long and narrow region, the lower part of a super-ellipsoid as depicted in Fig. 5(a) is fitted to describe the regular workspace expressed below:

$$
\frac{|x|^{2.5}}{385}+\frac{|y|^{2.5}}{470}+\frac{|z+320|^{2.5}}{400}=1
$$

\footnotetext{
${ }^{1}|\mathbf{M}|$ stands for the determinant of matrix $\mathbf{M}$
} 


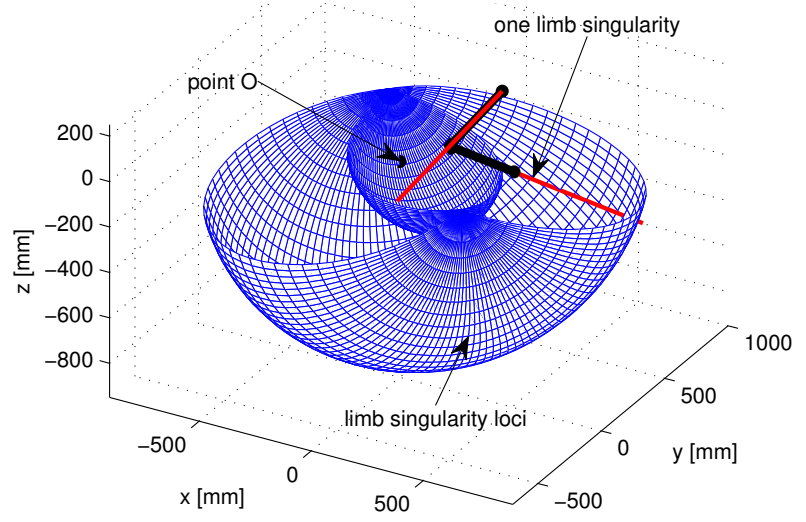

(a)

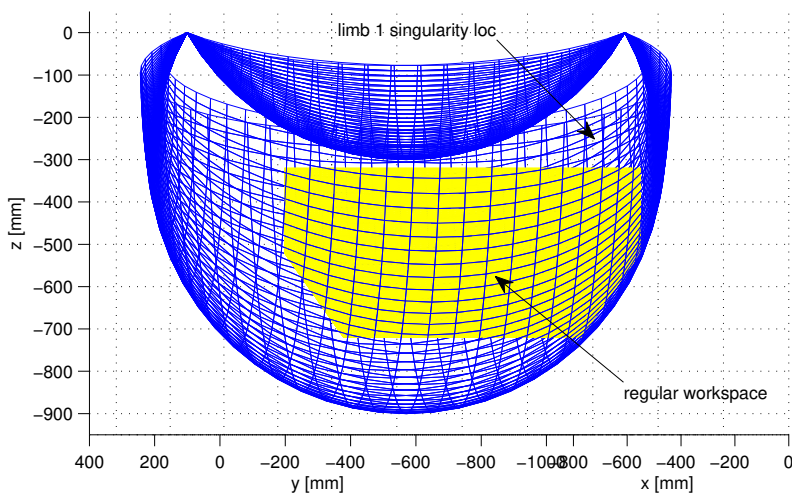

(b)

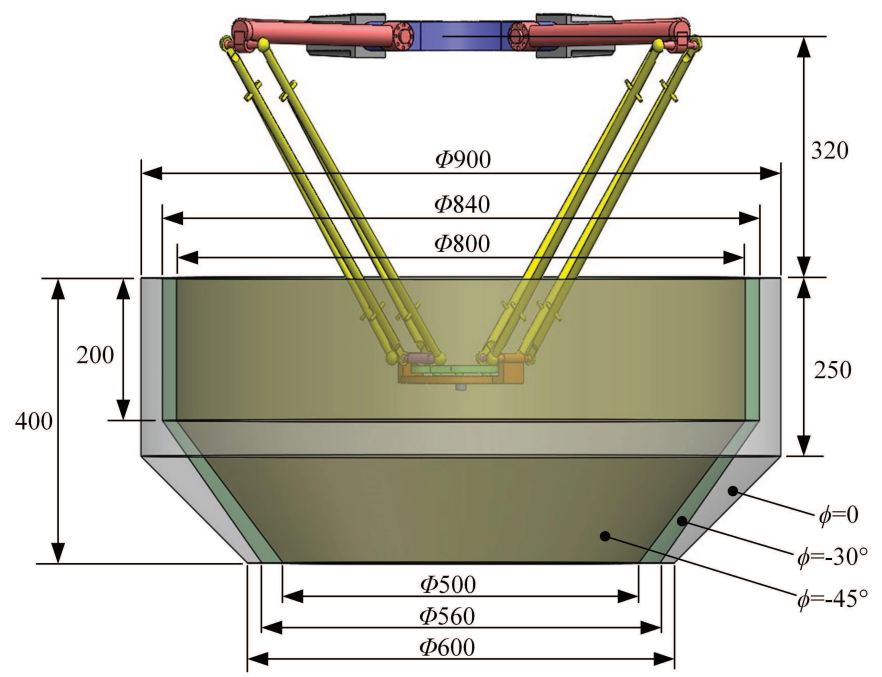

(c)

Figure 4: (a) Type-1 singularity loci and (b) maximum cylindrical workspace with constant endeffector orientation $\phi=0 \mathrm{rad}$; (c) regular workspace with different orientations. 


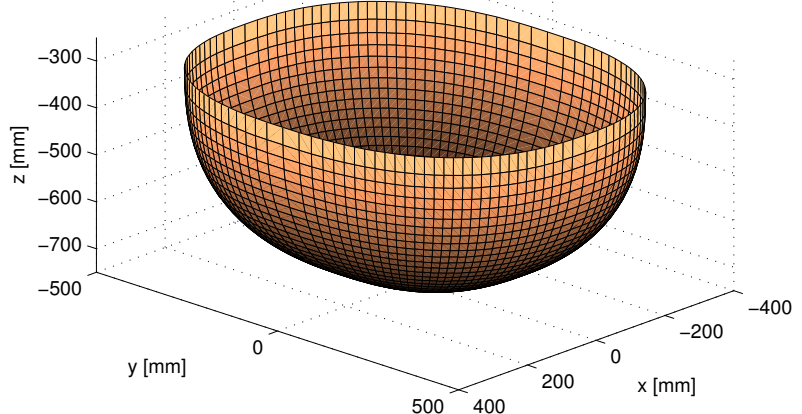

(a)

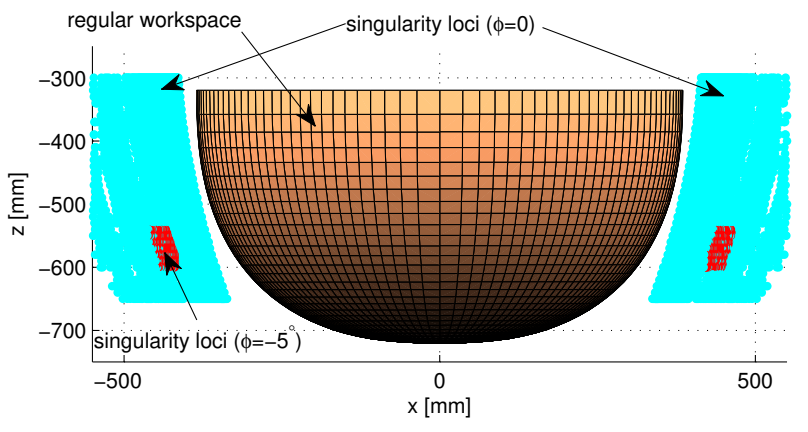

(b)

Figure 5: Type-2 singularity loci and the fitted super-ellipsoidal workspace.

thus, the robot admits a rectangular workspace free of Type- 2 singularity when the end-effector orientation is in the range of 0 and $-45^{\circ}$.

\subsubsection{Constraint singularity}

Besides the previous two type of singularities, the constraint singularity may exist within the workspace. In accordance with the singularity analysis of this four-limb parallel SMG [33,34], the constraint singularity usually occurs when at least two of the parallelograms are parallel or the rotational sub-platform lies in the plane determined by the two parallelograms in the first and third limbs [9]:

- From $|\mathbf{A}|=r \mathbf{s}^{T}\left(\mathbf{w}_{1} \otimes \mathbf{w}_{3}+\mathbf{w}_{3} \otimes \mathbf{w}_{1}\right)\left(\mathbf{w}_{2} \times \mathbf{w}_{4}\right)=0^{2}$, one constraint singularity occurs when $\mathbf{w}_{2} \times \mathbf{w}_{4}=\mathbf{0}$. It is noted that the segments $\overline{A_{2} B_{2}}$ and $\overline{A_{4} B_{4}}$ are always located in two parallel and noncoplanar planes that are both intersected with the segments $\overline{C_{2} C_{4}}$, which implies that this singular configuration cannot occur from practical viewpoint.

- When the robot is in a singular configuration of $\mathbf{w}_{1} \| \mathbf{w}_{2}$, as shown in Fig. 6(a), the robot has extra mobilities even though all the actuated joints are locked. Other singular configurations, for instance, $\mathbf{w}_{3}\left\|\mathbf{w}_{4}, \mathbf{w}_{1}\right\| \mathbf{w}_{3}$, and $\mathbf{w}_{1} \| \mathbf{w}_{4}\left(\mathbf{w}_{2} \| \mathbf{w}_{3}\right)$, are similar to this case.

- When the segment $C_{1} C_{3}$ lies in the plane determined by the vectors $\mathbf{w}_{1}$ and $\mathbf{w}_{3}$, as shown in Fig. 6(b), a screw motion with the axis coincident with $z$-axis will be unconstrained, thus, the robot has extra mobilities.

Constraint singularity loci The robot encounters the constraint singularity when it is in the configuration of $\mathbf{w}_{1} \| \mathbf{w}_{2}$, leading to

$$
\mathbf{w}_{1}-\mathbf{w}_{2}=\frac{1}{2}\left[\begin{array}{c}
\frac{1}{3} \cos \left(\phi_{0}+\phi\right)-\frac{\sqrt{2}}{3}-\frac{\sqrt{2}}{2} \cos \theta_{1}-\frac{\sqrt{2}}{2} \cos \theta_{2} \\
\frac{1}{3} \sin \left(\phi_{0}+\phi\right)-\frac{\sqrt{2}}{6}-\frac{\sqrt{2}}{2} \cos \theta_{1}+\frac{\sqrt{2}}{2} \cos \theta_{2} \\
\sin \theta_{1}-\sin \theta_{2}
\end{array}\right]=\mathbf{0}
$$

with the multiple solutions below

$$
\theta_{1}=\theta_{2}=\pi \pm \cos ^{-1}\left(\frac{1}{6}\right), \phi=0 \quad \text { or } \quad \theta_{1}=\theta_{2}= \pm \frac{2}{3} \pi, \phi=\frac{\pi}{2}
$$

\footnotetext{
${ }^{2} \otimes$ stands for tensor product of two vectors
} 

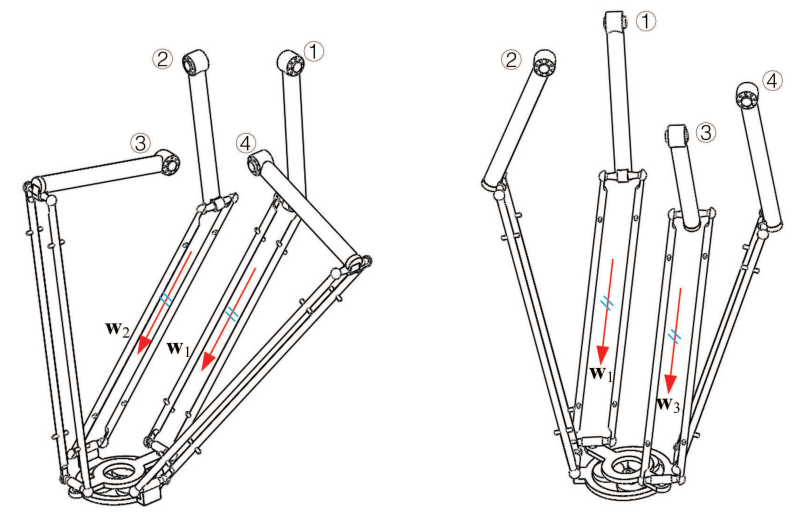

(a)
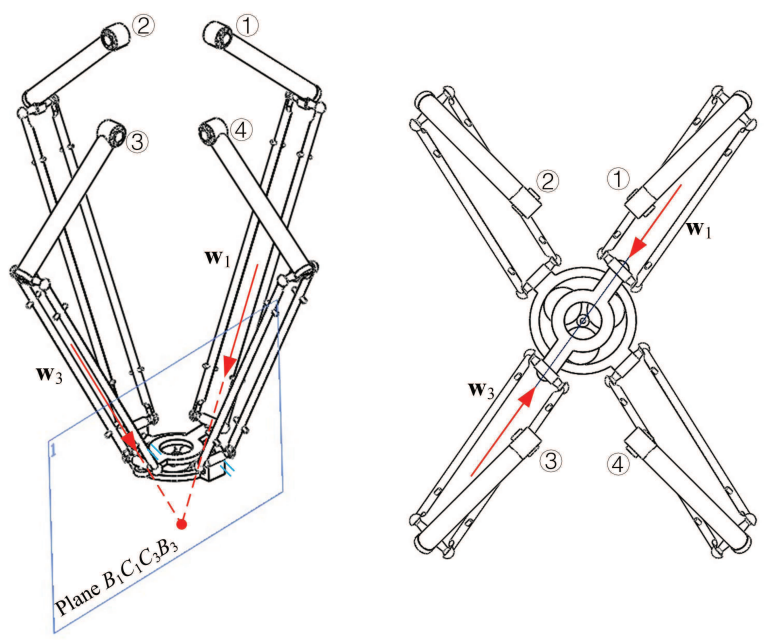

(b)

Figure 6: Constraint singularities of the SMG: (a) $\mathbf{w}_{1} \| \mathbf{w}_{2}$ and $\mathbf{w}_{1} \| \mathbf{w}_{3}$; (b) coplanar $\mathbf{w}_{1}$, $\mathbf{w}_{3}$ and $\overline{C_{1} C_{3}}$.

When the robot is in the configuration of $\phi=\pi / 2$ or $\theta_{1}=\theta_{2}=\pi+\cos ^{-1}(1 / 6)$, mechanical collisions occur. Consequently, substituting $\theta_{1}=\theta_{2}=\pi-\cos ^{-1}(1 / 6)$ into the first two kinematic constraint equations (9), the singularity loci are located on a spherical surface expressed as

$$
x^{2}+\left(y-a-\frac{\sqrt{2}}{2} R-\frac{\sqrt{2}}{2} b \cos \theta\right)^{2}+(z+b \sin \theta)^{2}=l^{2} ; \theta=\pi-\cos ^{-1}\left(\frac{1}{6}\right)
$$

for which the singularity loci are shown depicted in Fig. 7 together with the singularity loci in the configuration of $\mathbf{w}_{3} \| \mathbf{w}_{4}$. The singularity loci in these two cases are symmetrical with respect to $x z$ plane due to the symmetrical architecture of the robot.

Figure 8 shows the constraint singularity in the configuration $\mathbf{w}_{1} \| \mathbf{w}_{3}$, yielding

$$
\mathbf{w}_{1}-\mathbf{w}_{3}=\left[\begin{array}{c}
\frac{1}{3} \cos \left(\phi_{0}+\phi\right)-\frac{\sqrt{2}}{4}-\frac{\sqrt{2}}{4} \cos \theta_{1}-\frac{\sqrt{2}}{4} \cos \theta_{3} \\
\frac{1}{3} \sin \left(\phi_{0}+\phi\right)-\frac{\sqrt{2}}{4}-\frac{\sqrt{2}}{4} \cos \theta_{1}-\frac{\sqrt{2}}{4} \cos \theta_{3}-\frac{1}{3} \\
\frac{1}{2}\left(\sin \theta_{1}-\sin \theta_{3}\right)
\end{array}\right]=\mathbf{0}
$$

with the solutions below

$$
\theta_{1}=\theta_{3}= \pm \frac{2 \pi}{3}, \phi=\frac{\pi}{4} ; \theta_{1}=\theta_{3}=\pi \pm \cos ^{-1}\left(\frac{2}{3}+\frac{\sqrt{2}}{2}\right), \phi=\frac{3}{4} \pi
$$

To avoid the mechanical collisions, the singularity loci are located on a spherical surface by substituting $\theta_{1}=\theta_{3}=2 \pi / 3$ into the kinematic constraint equations (9), expressed as

$$
x^{2}+y^{2}+\left(z+b \sin \frac{2 \pi}{3}\right)^{2}=l^{2}
$$

Figure 9 shows the constraint singularity in the configuration $\mathbf{w}_{1} \| \mathbf{w}_{4}$, resulting in

$$
\mathbf{w}_{1}-\mathbf{w}_{4}=\frac{1}{2}\left[\begin{array}{c}
\frac{1}{3} \cos \left(\phi_{0}+\phi\right)-\frac{\sqrt{2}}{6}-\frac{\sqrt{2}}{2} \cos \theta_{1}+\frac{\sqrt{2}}{2} \cos \theta_{4} \\
\frac{1}{3} \sin \left(\phi_{0}+\phi\right)-\frac{\sqrt{2}}{3}-\frac{\sqrt{2}}{2} \cos \theta_{1}-\frac{\sqrt{2}}{2} \cos \theta_{4}-\frac{1}{6} \\
\sin \theta_{1}-\sin \theta_{4}
\end{array}\right]=\mathbf{0}
$$




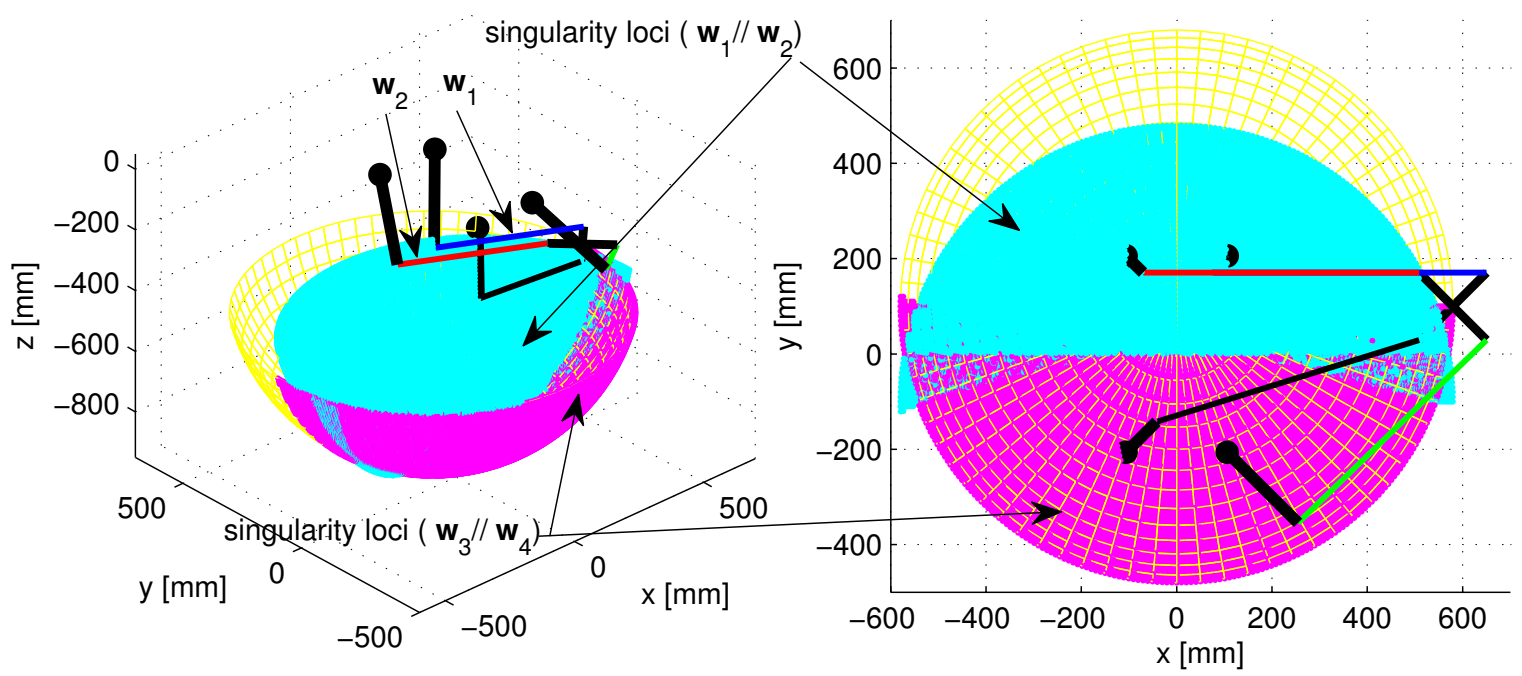

Figure 7: The singularity loci of the robot in the configurations of $\mathbf{w}_{1} \| \mathbf{w}_{2}\left(\mathbf{w}_{3} \| \mathbf{w}_{4}\right)$.

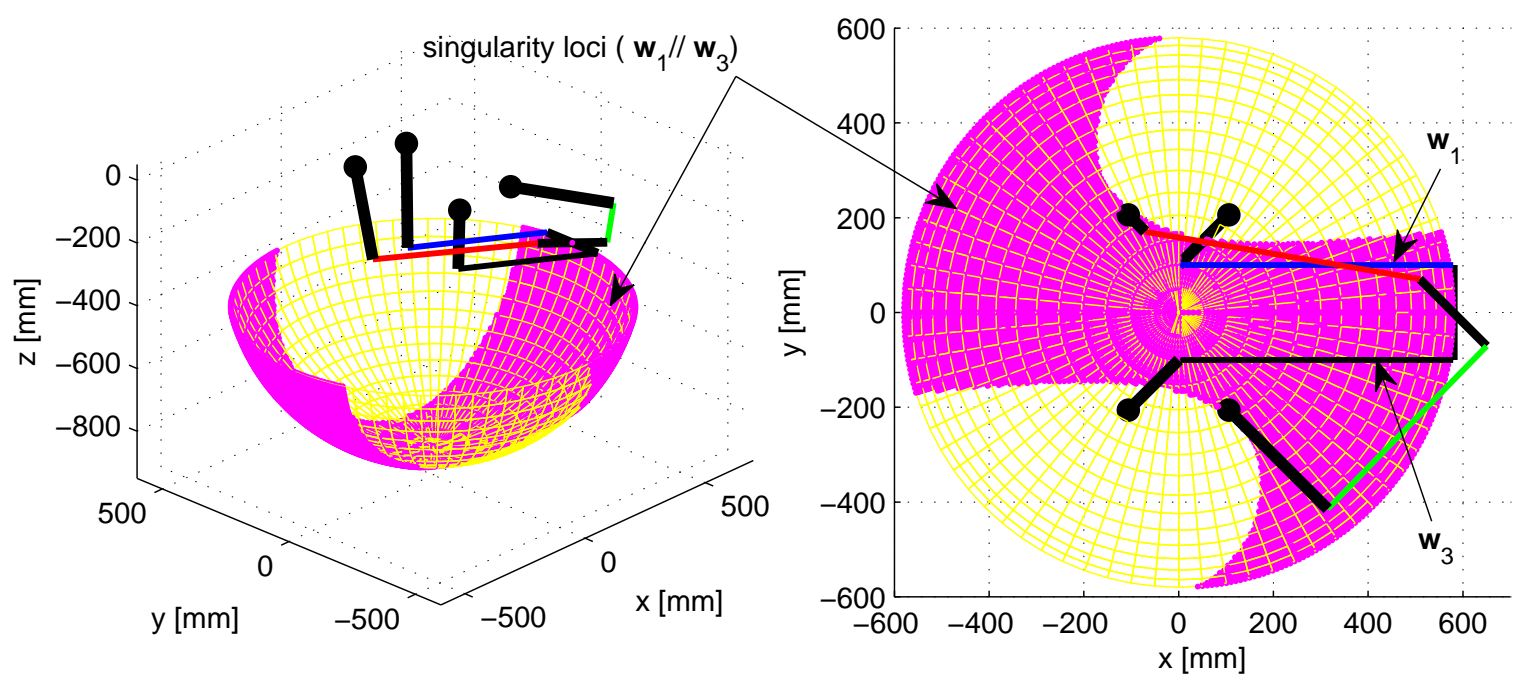

Figure 8: The singularity loci of the robot in the configurations of $\mathbf{w}_{1} \| \mathbf{w}_{3}$. 


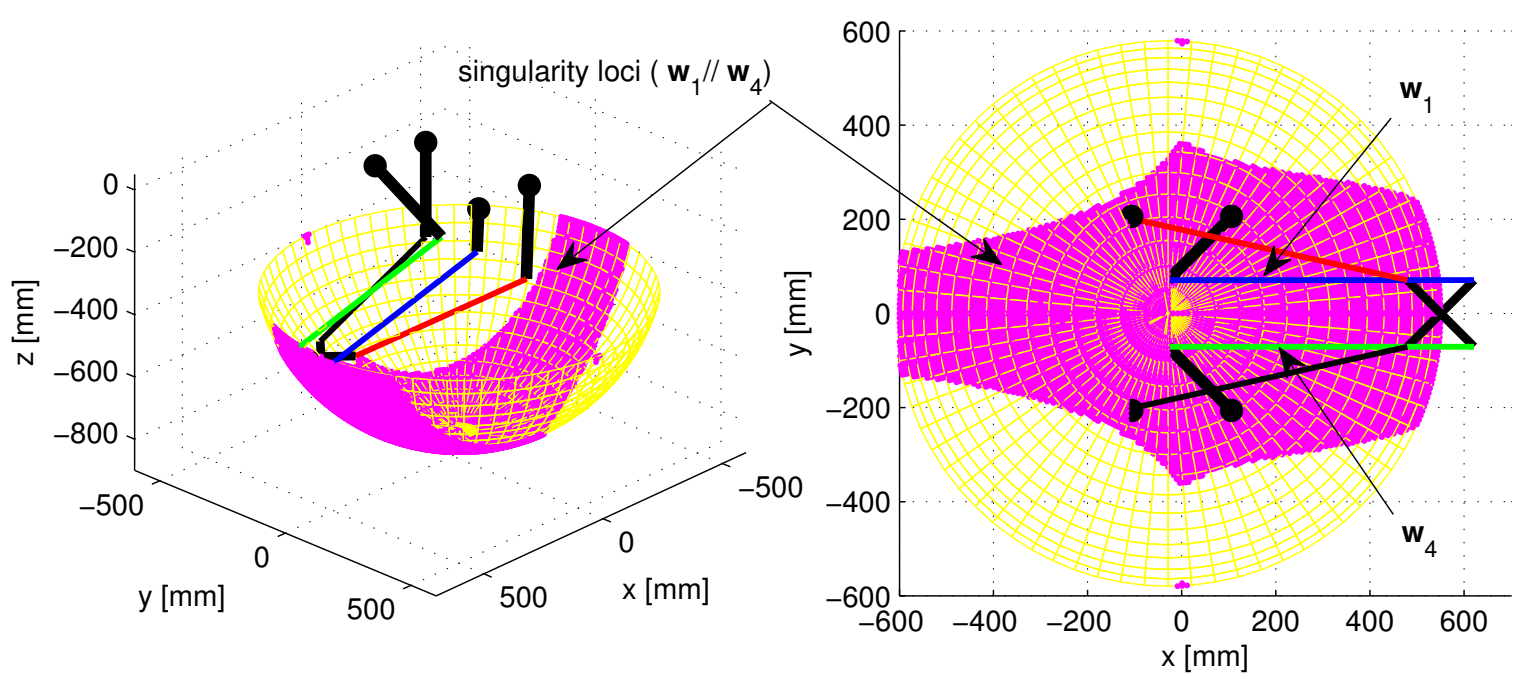

Figure 9: The singularity loci of the robot in the configurations of $\mathbf{w}_{1} \| \mathbf{w}_{4}$.

with the solutions below

$$
\theta_{1}=\theta_{2}=\pi \pm \cos ^{-1}\left(\frac{\sqrt{2}}{3}+\frac{1}{6}\right), \phi=0 ; \theta_{1}=\theta_{2}=\pi \pm \cos ^{-1}\left(\frac{\sqrt{2}}{3}+\frac{1}{2}\right), \phi=-\frac{\pi}{2}
$$

By the same token, to avoid the mechanical collisions, the singularity loci are located on a spherical surface expressed as

$$
\left(x-\frac{\sqrt{2}}{2} R-\frac{\sqrt{2}}{2} b \cos \theta\right)^{2}+y^{2}+(z+b \sin \theta)^{2}=l^{2} ; \theta=\pi-\cos ^{-1}\left(\frac{\sqrt{2}}{3}+\frac{1}{6}\right)
$$

The singularity loci with $\mathbf{w}_{2} \| \mathbf{w}_{3}$ are symmetrical to those with $\mathbf{w}_{1} \| \mathbf{w}_{4}$ with respect to $y z$ plane.

It is noteworthy that the robot encounters the previous constraint singularities with the corresponding limbs in posture of -, which can be avoided by keeping the robot limb working in posture of + through control algorithm.

When the robot encounters constraint singularity with the segment $\overline{C_{1} C_{3}}$ lying in the plane determined by the vectors $\mathbf{w}_{1}$ and $\mathbf{w}_{3}$, namely, points $B_{1}, B_{3}, C_{1}$ and $C_{3}$ being coplanar, the following geometric relationship exists

$$
\phi=\tan ^{-1} \frac{b_{1 y}-b_{3 y}}{b_{1 x}-b_{3 x}}-\frac{\pi}{4} ; \mathbf{w}_{1} \times \mathbf{w}_{3} \times \mathbf{s}=\mathbf{0}
$$

where $b_{i x}$ and $b_{i y}$ are the $x$ - and $y$-coordinates in the position vector $\mathbf{b}_{i}$ of Eq. (7), sequentially, the robot end-effector orientation is solved as

$$
\phi=\tan ^{-1} \frac{2 \sqrt{2} a+2 R+b \cos \theta_{1}+b \cos \theta_{3}}{2 R+b \cos \theta_{1}+b \cos \theta_{3}}-\frac{\pi}{4}
$$

then, the end-effector position can be readily solved according to the geometric configuration. The endeffector orientation with respect to the actuated joints $\theta_{1}$ and $\theta_{3}$ and the corresponding singularity loci are shown in Fig. 10. One observation from Fig. 10(a) is that the constraint loci in those configurations exists when the end-effector orientation $\phi$ is positive. This means that the constraint singularities will 


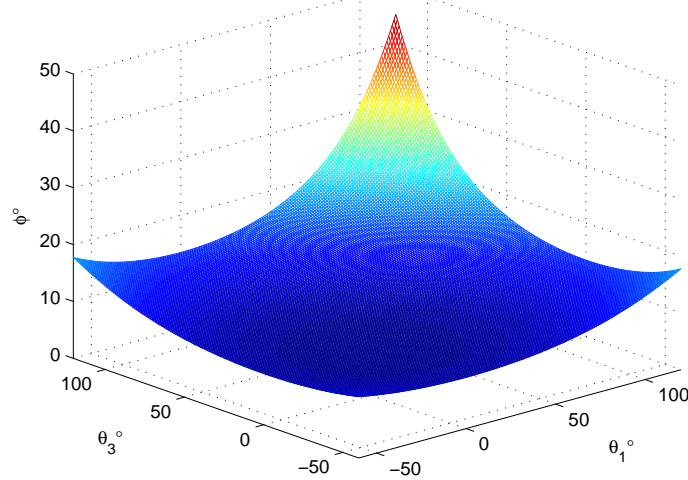

(a)

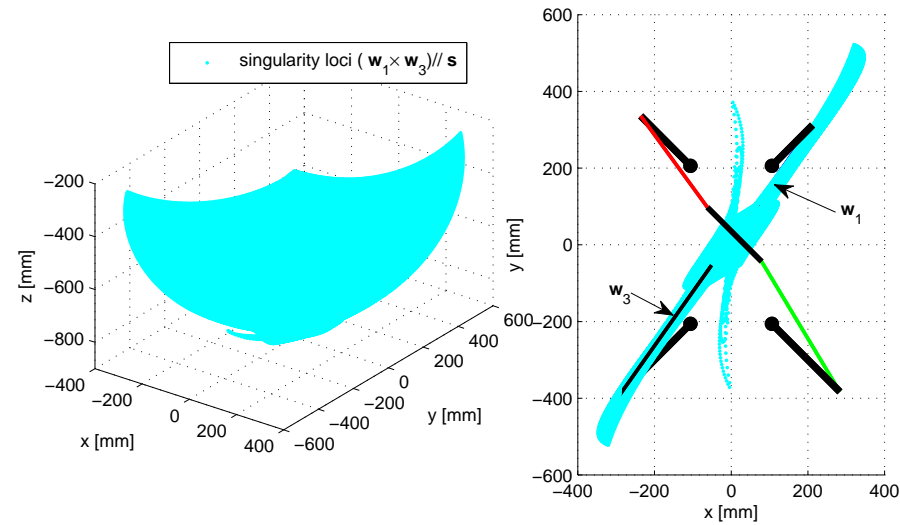

(b)

Figure 10: Constraint singularity with the segment $\overline{C_{1} C_{3}}$ lying in the plane determined by the vectors $\mathbf{w}_{1}$ and $\mathbf{w}_{3}$ : (a) end-effector orientation $\phi$ with the actuated joints $\theta_{1}$ and $\theta_{3}$; (b) singularity loci and top view of one singular configuration.

not occur as long as the rotational sub-platform rotates clockwise from the home orientation of the end-effector.

Based upon the foregoing singularity analysis, this robot admits a regular workspace in the shape of super-ellipsoid defined in Eq. (15), with the rotational sub-platform orientation $\phi \in\left[-45^{\circ}, 0\right]$ and the full-circle rotation of the end-effector, under first working mode.

\subsection{Dexterity over the singularity-free workspace}

Dexterity is another utmost important concern, which is usually evaluated by the condition number of the kinematic Jacobian matrix [35]. Since the elements in the forward Jacobian matrix A in Eq. (12) of the robot are not homogeneous due to both the translational and rotational motions of the end-effector, the following characteristic length [36] is applied for the normalization:

$$
L_{c}=\sqrt{\frac{3 \mathbf{J}_{\omega}^{T} \mathbf{J}_{\omega}}{\operatorname{tr}\left(\mathbf{J}_{v}^{T} \mathbf{J}_{v}\right)}}
$$

where $\mathbf{J}_{v}$ is the entry of the first three columns and $\mathbf{J}_{\omega}$ is the last one in $\mathbf{A}$, respectively. Sequentially, the normalized kinematic Jacobian matrix $\mathbf{J}_{N}=\mathbf{A}_{N}^{-1} \mathbf{B}$, based upon the normalized forward Jacobian $\mathbf{A}_{N}=\left[\begin{array}{ll}\mathbf{J}_{v} / L_{c} & \mathbf{J}_{\omega}\end{array}\right]$, is used to calculate the conditioning index of the dexterity with 2-norm. Thus, the characteristic length $L_{c}$ is derived when $\mathbf{A}_{N}^{T} \mathbf{A}_{N}$ have the four identical singular values, namely, the isotropy condition.

The predefined dexterity index is related to the kinematic isotropy of the robotic manipulator. The distributions of the normalized local condition index (LCI) over the different workspace cross-sections are depicted in Fig. 11. It can be seen from these figures that that the larger the magnitudes of the $z$ coordinate and of the orientation angle of the end-effector, the higher the dexterity index. The closer the end-effector to the workspace boundaries, the lower the dexterity index. Unlike the Quattro or Veloce. robots, the performance indices form asymmetric isocontours. 

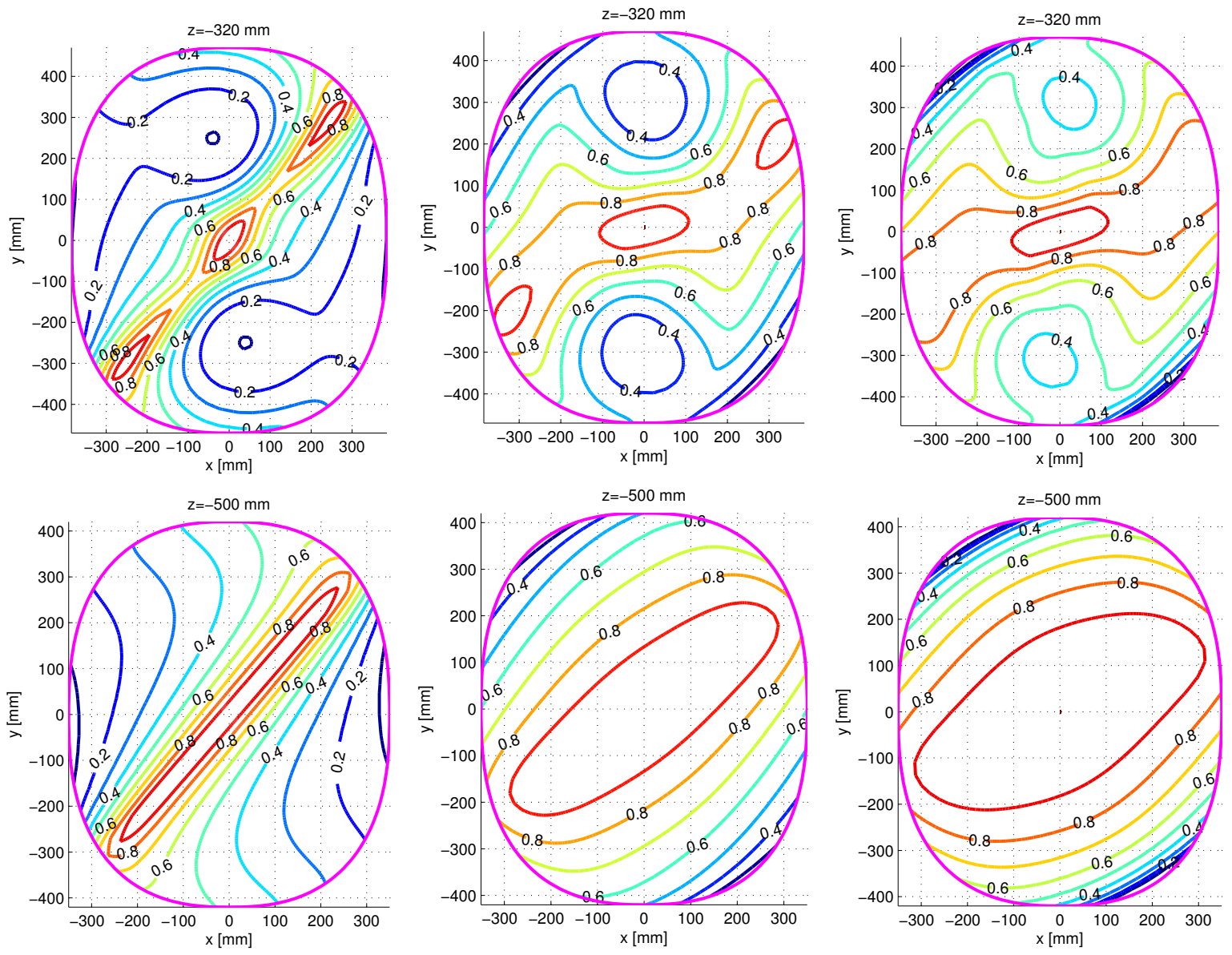

(a)

(b)

(c)

Figure 11: Isocontours of the dexterity index over different workspace cross-sections with constant end-effector orientation: (a) $\phi=0$; (b) $\phi=-30^{\circ}$; (c) $\phi=-45^{\circ}$; . 
Table 1: The mass and inertia properties of the robot links.

\begin{tabular}{lll}
\hline Terms & meanings & value \\
\hline$m_{b}$ & mass of the proximal arm & $0.5 \mathrm{~kg}$ \\
$I_{b}$ & moment of inertia of the proximal arm & $0.005 \mathrm{~kg} \cdot \mathrm{m}^{2}$ \\
$m_{l}$ & mass of a pairs of parallelogram & $0.2 \mathrm{~kg}$ \\
$I_{l}$ & moment of inertia of a pairs of parallelogram around point $B_{i}$ & $0.009 \mathrm{~kg} \cdot \mathrm{m}^{2}$ \\
$m_{p}$ & mass of the entire end-effector and payload & $3 \mathrm{~kg}$ \\
$I_{e x}$ & moment of inertia of the payload & $0.0012 \mathrm{~kg} \cdot \mathrm{m}^{2}$ \\
$m_{j}$ & mass of the connecting bar of parallelogram in limbs 1 and 3 & $0.03 \mathrm{~kg}$ \\
$I_{o}$ & moment of inertia of the output sun gear & $0.00001 \mathrm{~kg} \cdot \mathrm{m}^{2}$ \\
$I_{c}$ & moment of inertia of the carrier & $0.0025 \mathrm{~kg} \cdot \mathrm{m}^{2}$ \\
$I_{g}$ & moment of inertia of the planetary gear & $0.00003 \mathrm{~kg} \cdot \mathrm{m}^{2}$ \\
\hline
\end{tabular}

\subsection{Robot dynamics}

Robot dynamics of the SMG can be solved with the Lagrange equations [11,37], namely,

$$
\frac{\mathrm{d}}{\mathrm{d} t}\left(\frac{\partial L}{\partial \dot{\mathbf{q}}}\right)-\frac{\partial L}{\partial \mathbf{q}}+\boldsymbol{\Phi}_{q}^{T} \boldsymbol{\lambda}=\mathbf{Q}_{e x}
$$

where $L \equiv T-V$ is the Lagrangian of the system, including the mobile platform and the four legs, and $\mathbf{q}=\left[\begin{array}{ll}\boldsymbol{\theta}^{T} & \chi^{T}\end{array}\right]^{T}, \boldsymbol{\chi}$ being the robot pose defined in Eq. (12). Moreover, $\mathbf{Q}_{e x}=\left[\boldsymbol{\tau}^{T}, \mathbf{0}\right]^{T} \in \mathbb{R}^{8}$ is the vector of external forces and vector $\boldsymbol{\tau}=\left[\tau_{1}, \tau_{2}, \tau_{3}, \tau_{4}\right]^{T}$ is the actuated joint torque vector. Matrix $\mathbf{\Phi}_{q}=\left[\begin{array}{ll}\mathbf{B} & -\mathbf{A}\end{array}\right]$ is the system's constraint Jacobian. Moreover, $\boldsymbol{\lambda}=\left[\lambda_{1}, \lambda_{2}, \lambda_{3}, \lambda_{4}\right]^{T}$ is a vector of Lagrange multipliers.

In the formulation, it is assumed that the centers of mass of the proximal and distal arms are coincident with their geometric centers. The kinetic and potential energies are calculated below:

$$
\begin{aligned}
T= & \frac{1}{2}\left[\sum_{i=1}^{4}\left(I_{b} \dot{\theta}_{i}^{2}+\frac{1}{4} m_{l}\left\|\dot{\mathbf{b}}_{i}+\dot{\mathbf{c}}_{i}\right\|^{2}+I_{l} \frac{\left\|\dot{\mathbf{b}}_{i}-\dot{\mathbf{c}}_{i}\right\|^{2}}{l^{2}}\right)+\dot{\chi}^{T} \mathbf{M}_{p} \dot{\boldsymbol{\chi}}\right. \\
& \left.+m_{j}\left(\dot{\mathbf{c}}_{1}^{T} \dot{\mathbf{c}}_{1}+\dot{\mathbf{c}}_{3}^{T} \dot{\mathbf{c}}_{3}\right)+\left(I_{o}+I_{e x}\right)\left(i_{e H} \dot{\phi}\right)^{2}+3 I_{g}\left(i_{g H} \dot{\phi}\right)^{2}\right] \\
V= & \sum_{i=1}^{4}\left[\frac{1}{2} m_{b} \mathbf{b}_{i}+\frac{1}{2} m_{l}\left(\mathbf{b}_{i}+\mathbf{c}_{i}\right)\right] \cdot \mathbf{g}+m_{j}\left(\mathbf{c}_{1}+\mathbf{c}_{3}\right) \cdot \mathbf{g}+m_{p} \mathbf{p} \cdot \mathbf{g}
\end{aligned}
$$

where $\mathbf{M}_{p}=\operatorname{diag}\left[\begin{array}{llll}m_{p} & m_{p} & m_{p} & I_{c}\end{array}\right]$ is the mass matrix of the end-effector, and $\dot{\mathbf{b}}_{i}$ and $\dot{\mathbf{c}}_{i}$ stand for the velocities of points $B_{i}$ and $C_{i}$, respectively, which can be calculated with known $\dot{\theta}_{i}$ and $\dot{\boldsymbol{\chi}}$. Moreover, $i_{g H}$ stands for the speed ratio between the carrier and planetary gear, and $\mathbf{g}=[0,0,-9.806]^{T}$. The remaining terms are interpreted in Table 1. This modeling procedure has been evaluated with an ADAMS model in the previous work [38].

With the dynamic equation (28) in connection with the second-order kinematic model obtained from the time-differentiation Eq. (12), inverse dynamics can be handled to calculate the torque, power, 

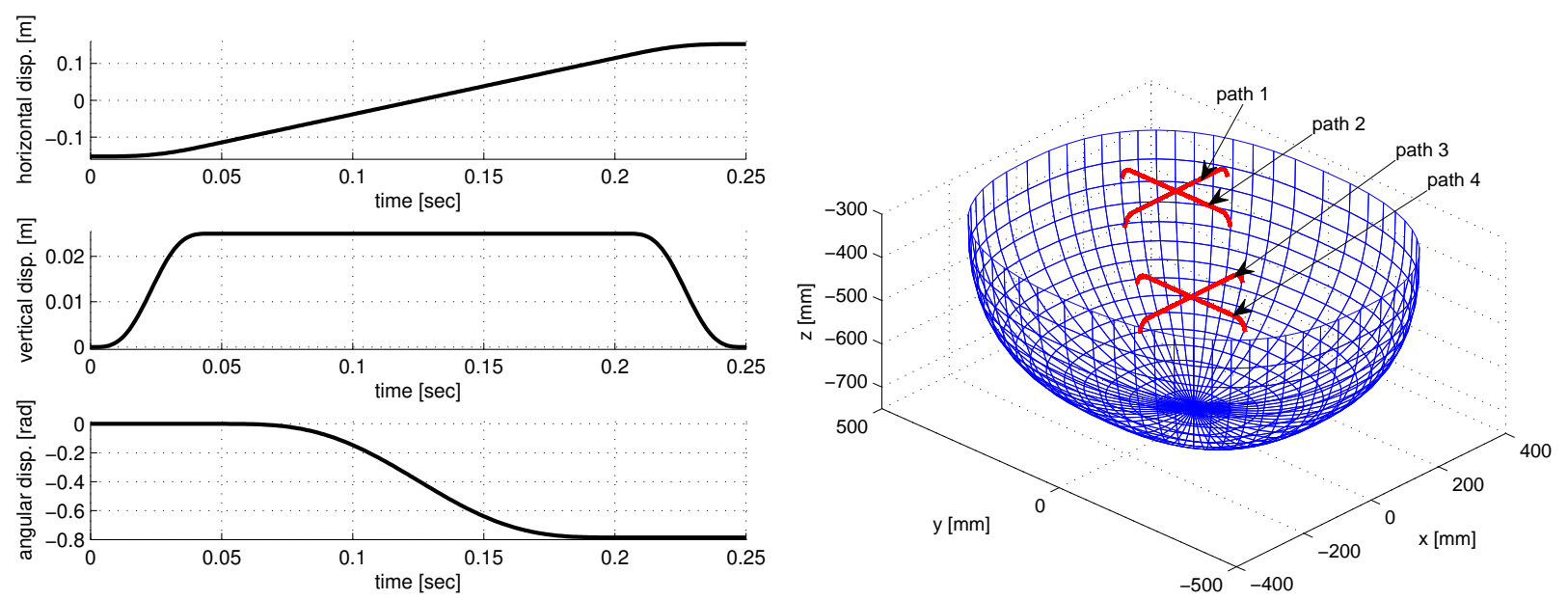

(a)

(b)

Figure 12: (a) Test trajectory of the pick-and-place operation and (b) paths within the workspace.

acceleration/velocity of the actuated joints. To evaluate the robot dynamics, simulation is carried out along an optimized test trajectory defined in [11], as displayed in Fig. 12(a). Here, the robot working cycle is set to 120 cycles per minute $(\mathrm{cpm})^{3}$, and four different paths within the workspace in Fig. 12(b) are selected, with the horizontal, vertical and angular displacements in unit of mm and rad, respectively, expressed as

$$
\begin{gathered}
h(t)=\left\{\begin{array}{l}
64.4 s(\tau)-152.5, \tau=10.8316 t ; \quad 0 \leq t \leq 0.046 \\
-120.3+120.3(t-0.046) / 0.0788 ; \quad 0.046<t \leq 0.203 \\
-64.4 s(\tau)+152.5, \tau=10.8316 t ; \quad 0.203<t \leq 0.250
\end{array}\right. \\
v(t)=\left\{\begin{array}{l}
25 s(\tau), \tau=21.6632 t ; \quad 0 \leq t \leq 0.046 \\
25 ; \quad 0.046<t \leq 0.203 \\
25 s(\tau), \tau=21.6632(0.250-t) ; \quad 0.203<t \leq 0.250
\end{array}\right. \\
\phi(t)= \begin{cases}0 ; \quad 0 \leq t \leq 0.046 \\
-\pi / 4 \cdot s(\tau), \tau=(t-0.046) /(t-0.092) ; \quad 0.046<t \leq 0.203 \\
-\pi / 4 ; \quad 0.203<t \leq 0.250\end{cases}
\end{gathered}
$$

where $s(\tau)=-20 \tau^{7}+70 \tau^{6}-84 \tau^{5}+35 \tau^{4}$ stands for the 4-5-6-7 polynomial equation.

Through comparison of the simulation results, the maximum torque of the actuated joints occurs in path 4 , and the maximum power consumption arises in path 1 . The corresponding simulation results along paths 1 and 4 are shown in Fig. 13, from which the specifications of the actuated joints can be summarized. For instance, the combination of a reduction gear box with speed ratio 30 and a motor with the constant speed $4000 \mathrm{rpm}$ and torque $6 \mathrm{Nm}$ can be selected to drive the proximal link, which can meet the dynamic requirements of the robot.

\subsection{Mechanical design and comparison with SMG counterparts}

One robot prototype is displayed in Fig. 14(a). The detailed mechanical design of the system is underway. The materials used include 1060 Alloy and FRP (Fiber Reinforced Polymer) composite.

\footnotetext{
${ }^{3} \mathrm{~A}$ cycle is defined as the round movement along a trajectory of $25 \times 305 \times 25 \mathrm{~mm}$ with a $1 \mathrm{~kg}$ payload in this work, of which the Adept test cycle is widely adopted for the smoothed trajectory [39] in many other cases.
} 

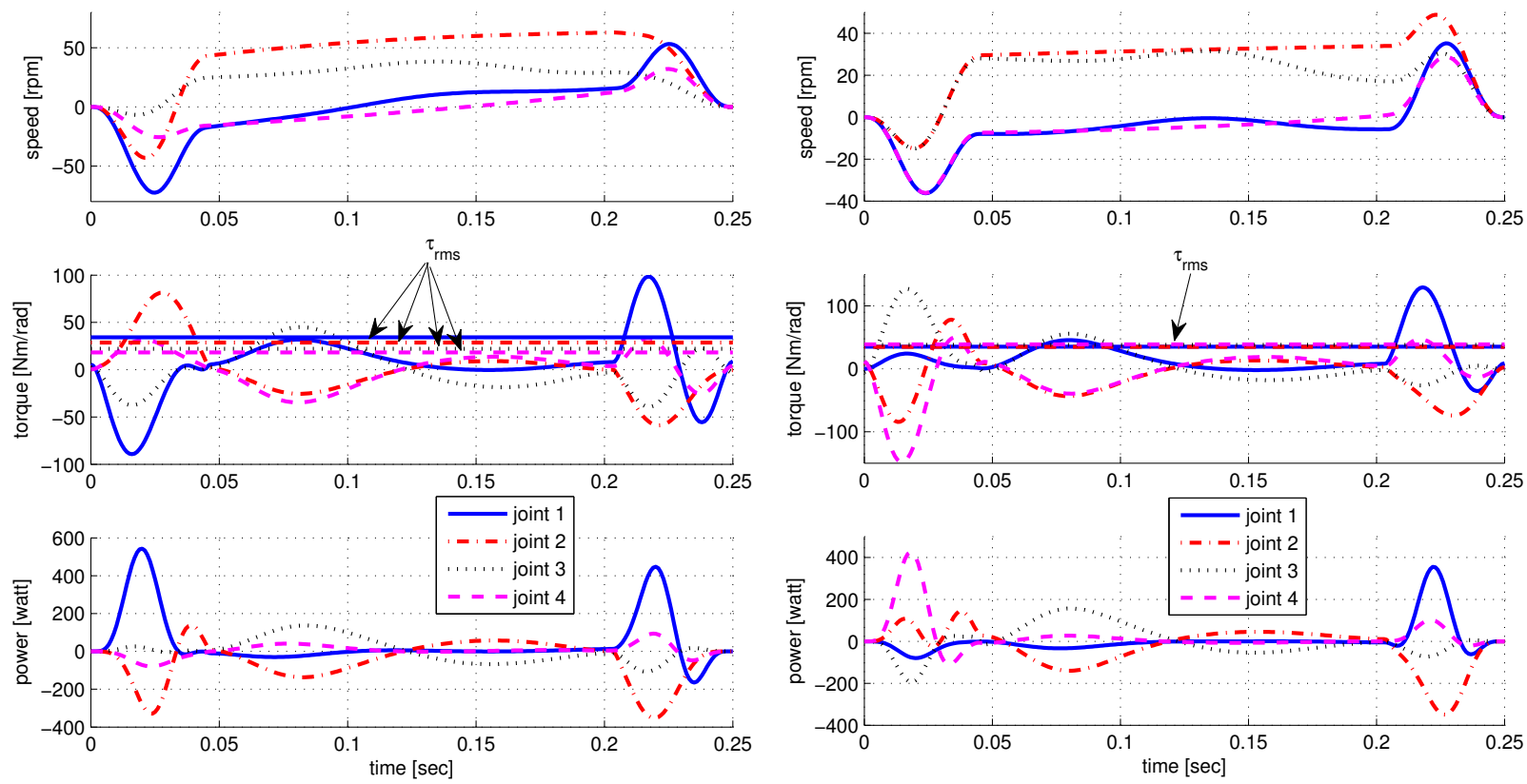

(a)

(b)

Figure 13: Simulation of robot dynamics: (a) path 1; (b) path 4.

Carbon fibre tubes are adopted to build the links together with 3D-printed components for lightweight design. Moreover, the bearing and gears are made of nylon polymer for the lightweight purpose, too. A simulation platform has been developed with V-Rep software, an open robot simulation environment, where the robot is integrated with sensors, visions and controllers for the purpose of demonstration and simulation. In the V-Rep environment, both mechanics and control of this robot can be simulated and analyzed.

Compared to the three-limb Delta robot [19] with an additional actuator on the end-effector or the fourth RPUR limb to connect the centers of the base and mobile platforms, which simplifies the robot architecture, four-limb SMGs can have larger workspace envelope. Moreover, as noted by Pierrot et al. [40], it is difficult to design a lightweight passive prismatic joint with a long service life. Another issue is that the use of $U$ joint as a transmitting mechanism suffering from the stability problem due to the nonlinear transmission [41], may induce vibrations and wear. The proposed end-effector can overcome the previous problems, as the gear train can transmit motion/force smoothly and efficiently.

From the perspective of workspace quality, the proposed robot can have a relatively large fullrotation workspace free of singularity versus the robot footprint, rather than some existing four-limb SMGs as revealed in our previous work [34]. Moreover, in comparison with the four-limb SMG end-effectors with different shape and size corresponding to different orientations, e.g., belt-pulley mechanism [4], the rack-pinion mechanism [3], gears and screw mechanism [5], an advantage is that the articulated transmitting mechanism can be sealed in the gearbox, which eases the cleaning procedure when the robot is deployed in the sector of food industry. On the other hand, the proposed end-effector composed of a planetary gear train has similar structural complexity to the foregoing ones.

\section{Conclusions}

This paper introduced a four-limb parallel Schönflies motion generator for pick-and-place (PnP) applications, whose end-effector adopts a planetary gear train as the amplification mechanism to realize 


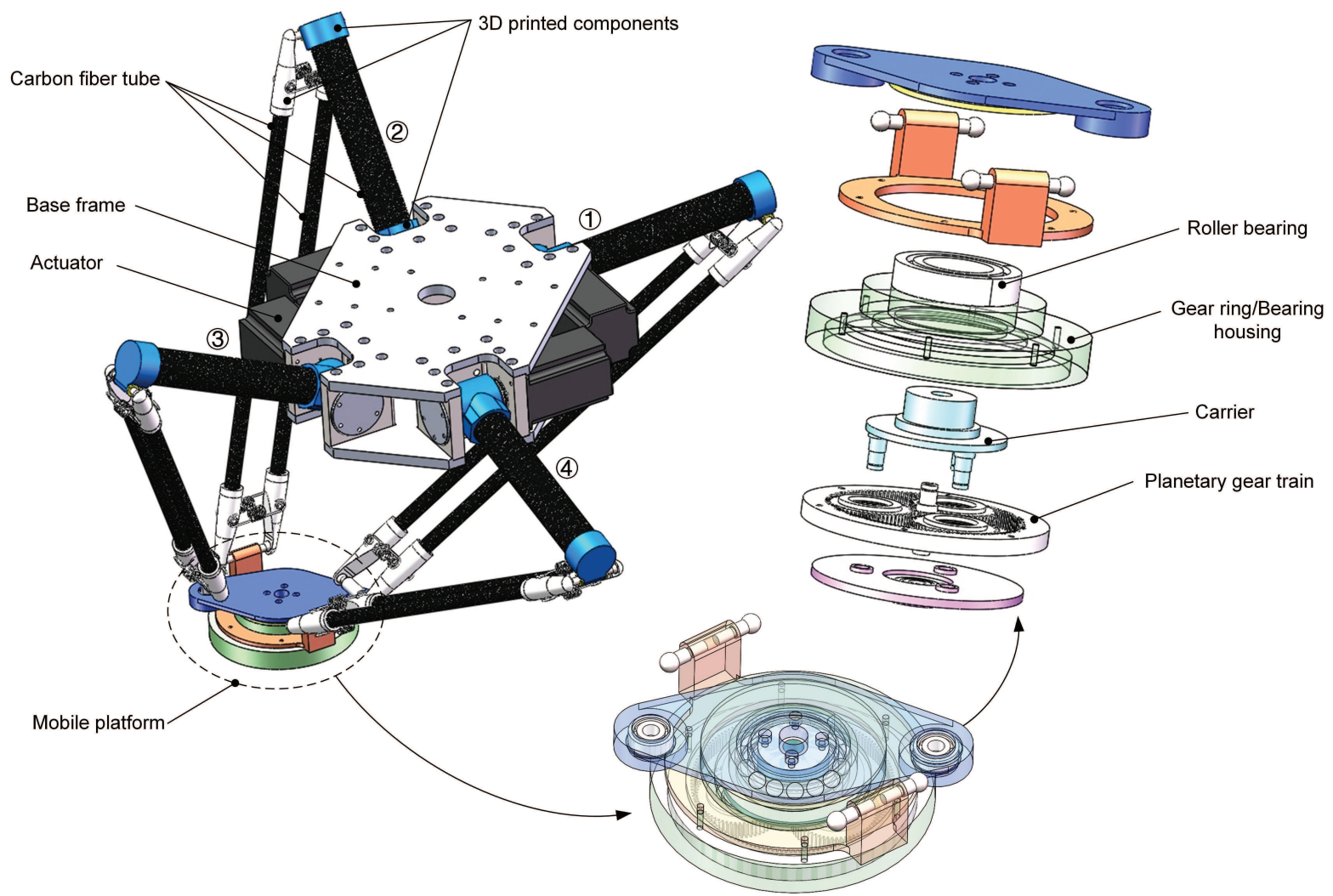

(a)
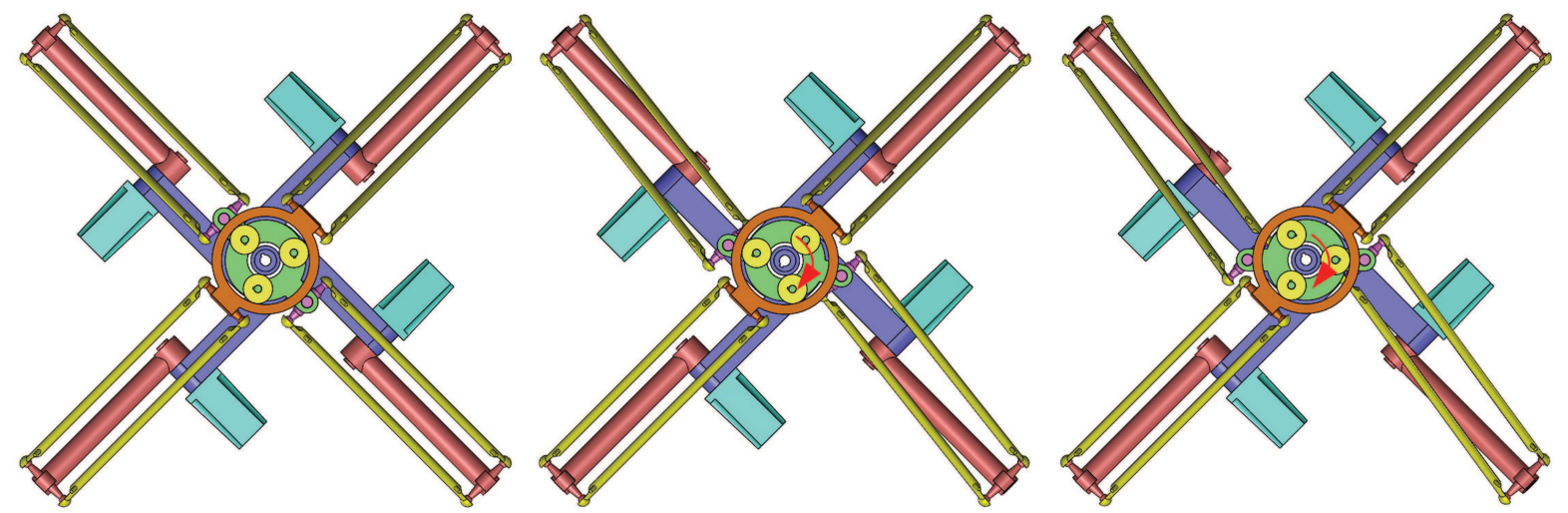

(b)

Figure 14: The CAD model of the four-limb SMG with planetary gear train: (a) rended model; (b) bottom view of the simplified end-effector with orientations $\phi=0, \phi=-30^{\circ}$ and $\phi=-45^{\circ}$ in order. 
the full-circle rotation. The motion type of the robot end-effector was examined by means of the Lie group theory. The kinematic aspects including the workspace, dexterity and singularity were analyzed and evaluated. The singular configurations and the singularity loci were identified both graphically and numerically, which shows that the singular configurations of the manipulator can be avoided by keeping all the limbs working in a prescribed working mode together with the end-effector rotation in a clockwise direction from the home orientation.

The dexterity evaluation is carried out to depict the workspace quality and dexterous working envelope. The dexterous workspace regions increase with the increasing distance between the mobile platform and the base as well as the increasing orientation angle of the end-effector. The regular workspace, namely, a long and narrow super-ellipsoidal workspace with the full-circle rotation of the end-effector, is identified under a given working mode without crossing any singularity, which turns out that the proposed robot is suitable for PnP operations. Moreover, robot dynamics was analyzed to to select the actuators of the good under design. Finally, comparison the proposed SMG manipulator to its SMG counterparts highlights the advantage of the proposed robot for food industry, mainly due to fact that the end-effector can be easily cleaned, while having a robot competitive in terms of workspace size, orientation range and dynamic performance.

\section{Acknowledgement}

The supports from the Fundamental Research Funds for the Central Universities (No.DUT19JC25), the Natural Science Foundation and the Doctoral Start up Foundation of Liaoning Province (Nos. 20180520028, 20170520134), and the Applied Basic Research Programs of Changzhou (No. CJ20180017) are gratefully acknowledged.

\section{References}

[1] F. Pierrot and O. Company. H4: a new family of 4-dof parallel robots. In IEEE/ASME Inter. Conf. Advan. Intell. Mecha., pages 508-513. IEEE, 1999.

[2] O. Company, S. Krut, and F. Pierrot. Internal singularity analysis of a class of lower mobility parallel manipulators with articulated traveling plate. IEEE Trans. Robot., 22(1):1-11, 2006.

[3] S. Krut, M. Benoit, H. Ota, and F. Pierrot. I4: A new parallel mechanism for SCARA motions. In IEEE Int. Conf. Robot. Autom., volume 2, pages 1875-1880. IEEE, 2003.

[4] V. Nabat, M. de la O Rodriguez, O. Company, S. Krut, and F. Pierrot. Par4: very high speed parallel robot for pick-and-place. In IEEE/RSJ Inter. Conf. Intell. Robot. Syst., pages 553-558. IEEE, 2005.

[5] D. Corbel, M. Gouttefarde, O. Company, and F. Pierrot. Actuation redundancy as a way to improve the acceleration capabilities of $3 \mathrm{~T}$ and $3 \mathrm{~T} 1 \mathrm{R}$ pick-and-place parallel manipulators. ASME J. Mech. Robot., 2(4), 2010.

[6] S. M. Kim, W. Kim, and B.-J. Yi. Kinematic analysis and optimal design of a 3T1R type parallel mechanism. In IEEE Int. Conf. Robot. Autom., pages 2199-2204, 2009.

[7] O. Altuzarra, B. Şandru, Ch. Pinto, and V. Petuya. A symmetric parallel Schönflies-motion manipulator for pick-and-place operations. Robotica, 29:853-862, 2011. 
[8] S. Liu, T. Huang, J. Mei, X. Zhao, P. Wang, and D. G Chetwynd. Optimal design of a 4-DOF SCARA type parallel robot using dynamic performance indices and angular constraints. ASME J. Mech. Robot., 4(3), 2012.

[9] F. Xie and X. Liu. Design and development of a high-speed and high-rotation robot with four identical arms and a single platform. ASME J. Mech. Robot., 7(4), 2015.

[10] G. Wu. Kinematic analysis and optimal design of a wall-mounted four-limb parallel Schönfliesmotion robot for pick-and-place operations. J. Intell. Robot. Syst., 85(3):663-677, 2017.

[11] G. Wu, S. Bai, and P. Hjørnet. Architecture optimization of a parallel Schönflies-motion robot for pick-and-place applications in a predefined workspace. Mech. Mach. Theory, 106:148-165, 2016.

[12] X. Kong and C. M. Gosselin. Type synthesis of 3T1R 4-dof parallel manipulators based on screw theory. IEEE Trans. Robot. Autom., 20(2):181-190, 2004.

[13] G. Gogu. Structural synthesis of fully-isotropic parallel robots with Schönflies motions via theory of linear transformations and evolutionary morphology. Euro. J. Mech.-A/Solids, 26(2):242-269, 2007.

[14] P.-L. Richard, C. M. Gosselin, and X. Kong. Kinematic analysis and prototyping of a partially decoupled 4-DOF 3T1R parallel manipulator. ASME J. Mech. Des., 129(6):611-616, 2007.

[15] Q. Li and J. M. Hervé. Parallel mechanisms with bifurcation of Schoenflies motion. IEEE Trans. Robot., 25(1):158-164, 2009.

[16] S. Briot, V. Arakelian, and S. Guégan. PAMINSA: A new family of partially decoupled parallel manipulators. Mech. Mach. Theory, 44(2):425-444, 2009.

[17] T.-L. Yang, A. Liu, H. Shen, L. Hang, Y. Luo, and Q. Jin. Topology Design of (3T-1R) Parallel Mechanisms, pages 207-236. Springer Singapore, 2018.

[18] H. Shen, Z. Xu, K. Xu, S. Bai, J. Deng, G. Wu, and T.-l. Yang. Design and direct position analysis of a new 3T1R parallel manipulator with low coupling degree. In B. Corves and M. Wenger, P.and Hüsing, editors, EuCoMeS 2018, pages 333-339, Cham, 2019. Springer International Publishing.

[19] F. Pierrot, C. Reynaud, and A. Fournier. DELTA: a simple and efficient parallel robot. Robotica, 8(2):105-109, 1990.

[20] J. Angeles, S. Caro, W. Khan, and A. Morozov. Kinetostatic design of an innovative Schönfliesmotion generator. Proc. Ins. Mech. Eng. Part C: J. Mech. Eng. Sci., 220(7):935-943, 2006.

[21] P.-C. Lee and J.-J. Lee. Singularity and workspace analysis of three isoconstrained parallel manipulators with Schoenflies motion. Front. Mech. Eng., 7(2):163-187, 2012.

[22] S. M. Kim, K. Shin, B.-J. Yi, and W. Kim. Development of a novel two-limbed parallel mechanism having Schönflies motion. Proc. Ins. Mech. Eng. Part C: J. Mech. Eng. Sci., 229(1):136-154, 2015.

[23] L. Xu, Q. Chen, L. He, and Q. Li. Kinematic analysis and design of a novel 3T1R 2-(PRR)2RH hybrid manipulator. Mech. Mach. Theory, 112:105-122, 2017.

[24] P. Karimi Eskandary and J. Angeles. The dynamics of a parallel Schönflies-motion generator. Mech. Mach. Theory, 119:119-129, 2018. 
[25] H. Simas and R. Di Gregorio. Position analysis, singularity loci and workspace of a novel 2PRPU schoenflies-motion generator. Robotica, 37(1):141-160, 2019.

[26] T. Harada and J. Angeles. Kinematics and singularity analysis of a CRRHHRRC parallel Schönflies motion generator. CSME Transactions, 38(2):173-183, 2014.

[27] C. Gosselin, M. Isaksson, K. Marlow, and T. Laliberte. Workspace and sensitivity analysis of a novel non-redundant parallel SCARA robot featuring infinite tool rotation. IEEE Robotics \& Automation Letters, 1(2):776-783, 2016.

[28] J. M. Hervé. The lie group of rigid body displacements, a fundamental tool for mechanism design. Mech. Mach. Theory, 34(5):719-730, 1999.

[29] G. Wu, Z. Lin, H. Shen, W. Zhao, and S. Zhang. A new four-limb parallel Schönflies motion generator with end-effector full-circle rotation via planetary gear train. Lecture Notes in Computer Science, 11742:425-435, 2019.

[30] C. Gosselin and J. Angeles. Singularity analysis of closed-loop kinematic chains. IEEE Trans. Robot. Autom., 6(3):281-290, 1990.

[31] D. Zlatanov, I. A. Bonev, and C. M. Gosselin. Constraint singularities of parallel mechanisms. In IEEE Int. Conf. Robot. Autom., volume 1, pages 496-502. IEEE, 2002.

[32] J.-P. Merlet. Singular configurations of parallel manipulators and Grassmann geometry. The Int. J. Robot. Res., 8(5):45-56, 1989.

[33] S. Amine, M. T. Masouleh, S. Caro, P. Wenger, and C. Gosselin. Singularity conditions of 3T1R parallel manipulators with identical limb structures. ASME J. Mech. Robot., 4(1):011011, 2012.

[34] G. Wu, S. Bai, and S. Caro. A transmission quality index for a class of four-limb parallel schönflies motion generators. ASME J. Mech. Robot., 10(5), 2018.

[35] C. Gosselin and J. Angeles. A global performance index for the kinematic optimization of robotic manipulators. ASME J. Mech. Des., 113(3):220-226, 1991.

[36] O. Altuzarra, A. Hernandez, O. Salgado, and J. Angeles. Multiobjective optimum design of a symmetric parallel Schönflies-motion generator. ASME J. Mech. Des., 131(3):031002, 2009.

[37] J. G. De Jalon and E. Bayo. Kinematic and Dynamic Simulation of Multibody Systems: the Real-time Challenge. Springer Science \& Business Media, 2012.

[38] G. Wu, S. Bai, and P. Hjørnet. Design analysis and dynamic modeling of a high-speed 3T1R pick-and-place parallel robot. In S Bai and M. Ceccarelli, editors, Mechanisms and Machine Science: Recent Advances in Mechanism Design for Robotics, volume 33, pages 285-295, 2015.

[39] J. F. Gauthier, J. Angeles, and S. Nokleby. Optimization of a test trajectory for SCARA systems. In J. Lenarčič and P. Wenger, editors, Advances in Robot Kinematics: Analysis and Design, pages 225-234. Springer Netherlands, Dordrecht, 2008.

[40] F. Pierrot, O. Company, S. Krut, and V. Nabat. Four-dof PKM with articulated travelling-plate. In Parallel Kinematics Seminar (PKS'06), pages 25-26, Chemnitz, Germany, Apr. 25-26 2006.

[41] R. M. Rosenberg. On the dynamical behavior of rotating shafts driven by universal (hooke) couplings. ASME J. Appl. Mech., 25(1):47-51, 1958. 